\title{
Taurine Attenuates Calpain-2 Induction and a Series of Cell Damage via Suppression of NOX-Derived ROS in ARPE-19 Cells
}

\author{
Yuanyuan Zhang $\mathbb{D}^{\mathbb{D}}$, Shu Ren $\left(\mathbb{D}\right.$, Yanting Gu, Jiahong Wang, Zheng Liu $\mathbb{D}^{\mathbb{D}}$, and Zhou Zhang \\ Department of Pharmacology, Shenyang Pharmaceutical University, Shenyang 110016, China \\ Correspondence should be addressed to Zheng Liu; zhengliu@syphu.edu.cn and Zhou Zhang; zhouzhang@syphu.edu.cn
}

Received 21 March 2018; Revised 24 May 2018; Accepted 7 June 2018; Published 29 July 2018

Academic Editor: Karolina Szewczyk-Golec

Copyright (C) 2018 Yuanyuan Zhang et al. This is an open access article distributed under the Creative Commons Attribution License, which permits unrestricted use, distribution, and reproduction in any medium, provided the original work is properly cited.

\begin{abstract}
Nicotinamide adenine dinucleotide phosphate (NADPH) oxidases (NOXs) are key transmembrane proteins leading to reactive oxygen species (ROS) overproduction. However, the detailed roles of NOXs in retinal pigment epithelial (RPE) cell metabolic stress induced by Earle's balanced salt solution (EBSS) through starvation remain unclear. In this study, we investigated what roles NOXs play in regard to calpain activity, endoplasmic stress (ER), autophagy, and apoptosis during metabolic stress in ARPE-19 cells. We first found that EBSS induced an increase in NOX2, NOX4, p22phox, and NOX5 compared to NOX1. Secondly, suppression of NOXs resulted in reduced ER stress and autophagy, decreased ROS generation, and alleviated cell apoptosis. Thirdly, silencing of NOX4, NOX5, and p22phox resulted in reduced levels of cell damage. However, silencing of NOX1 was unaffected. Finally, taurine critically mediated NOXs in response to EBSS stress. In conclusion, this study demonstrated for the first time that NOX oxidases are the upstream regulators of calpain-2, ER stress, autophagy, and apoptosis. Furthermore, the protective effect of taurine is mediated by the reduction of NOX-derived ROS, leading to sequential suppression of calpain induction, ER stress, autophagy, and apoptosis.
\end{abstract}

\section{Introduction}

Reactive oxygen species (ROS) are signaling molecules that result in metabolic stress, changes in mitochondrial membrane permeability, DNA damage, and cell apoptosis [1-3]. ROS are generated by many cell types in the human body and are involved in the pathogenesis of various ocular diseases $[4,5]$, including glaucoma [6], age-related macular degeneration (AMD) [7], and retinopathy [8-10].

Nicotinamide adenine dinucleotide phosphate (NADPH) oxidase (NOX) is another key source of ROS besides mitochondria $[11,12]$. NOX is the only enzyme group that produces ROS as its main function. Studies have shown that the NOX family is the inducer of ROS generation, ER stress, autophagy, and apoptosis $[1,13,14]$. There are different isoforms of NOXs in mammalian cells, containing NOX1-5 and DUOX1 and DUOX2 [15]. Of these homologues, we discovered that human retina expresses NOX1, NOX2, NOX4, and NOX5 [15-17]. Studies have shown that different homologues play different roles in retinal pathological processes. Some studies indicate that NOX1 is increased during eye disease and cardiac dysfunction [18, 19]. NOX2 is upregulated during ocular injury and diabetes $[4,16]$. The expression level of NOX4 is increased during cardiomyocyte injury, diabetic retinopathy, and stroke $[10,13,20]$. The p22phox subunit is an essential part of the NOX compound. Except for NOX5 and DUOX1/2, p22phox is required for regulating NOX isoforms [3, 21]. Unlike other NOX homologues, NOX5 has the ability to bind and be activated by intracellular calcium directly and its function to produce ROS is regulated by intracellular calcium mobilization, influx, and phosphorylation [22, 23]. Calcium/calmodulin-dependent kinase II can activate NOX5 via direct phosphorylation [15]. Moreover, some studies show that crosstalk between ROS and calpain leads to the release of $\mathrm{Ca}^{2+}$ [24-27]. The nuclear translocation of calpain-2 can be activated by increased NOX- 

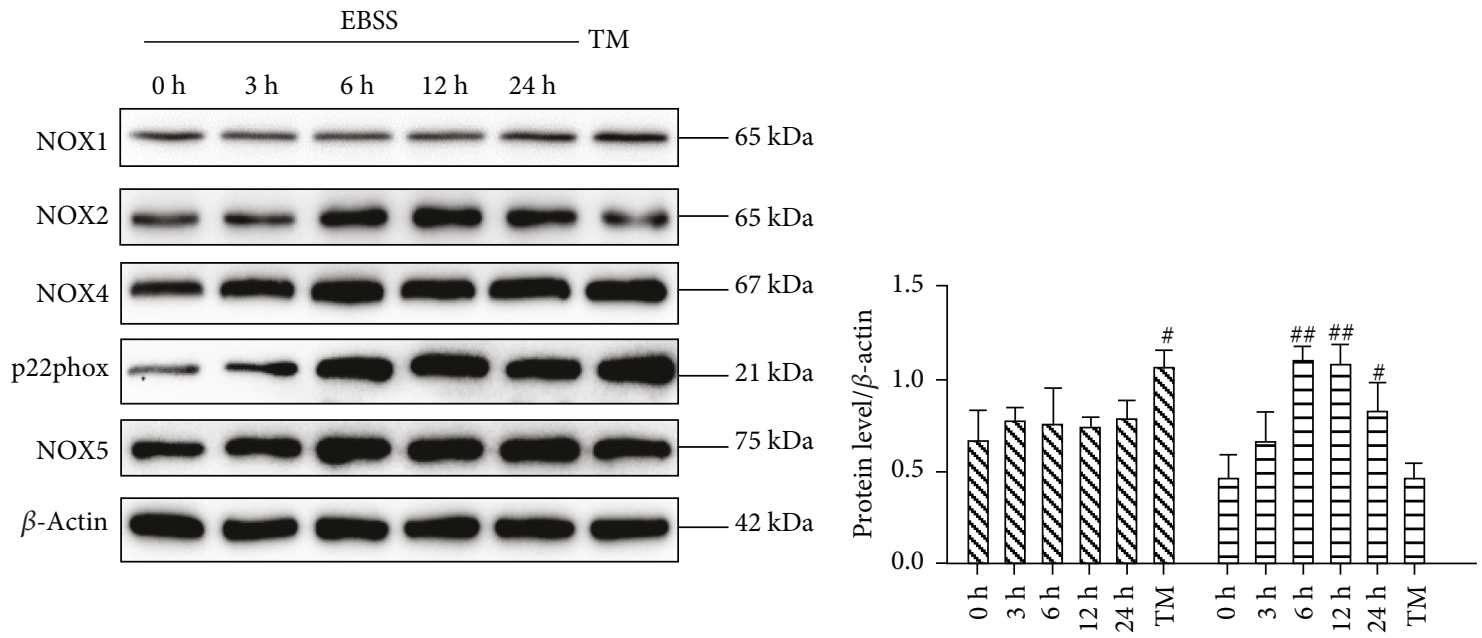

5. NOX1

$\boxminus \mathrm{NOX} 2$

(a)

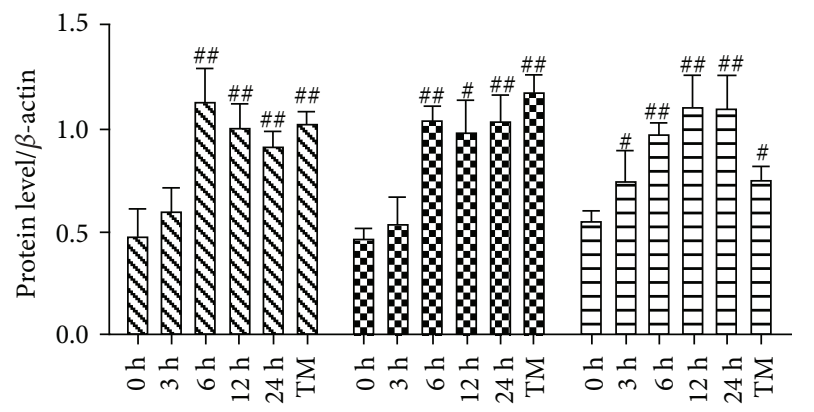

$\$ \mathrm{NOX} 4$

nes 22phox

$\boxminus \operatorname{NOX} 5$

(c)
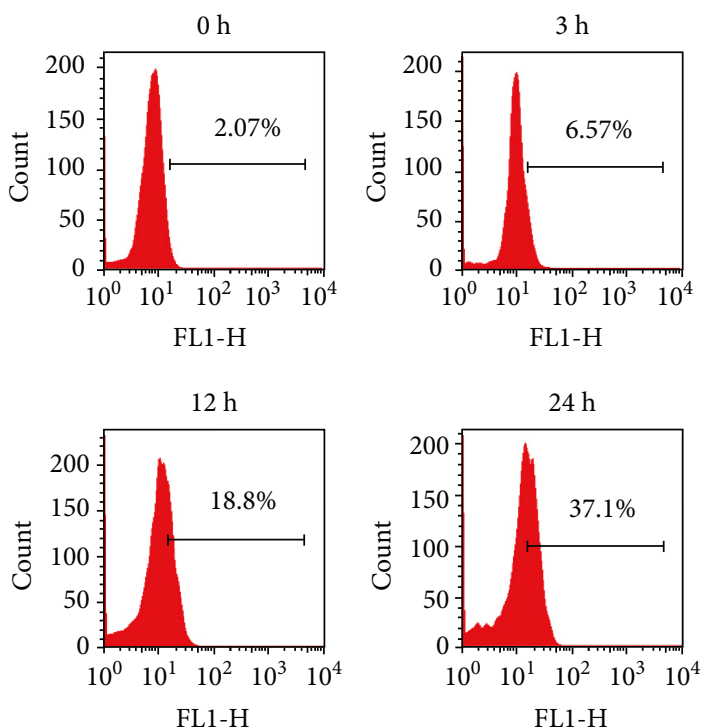

(d)
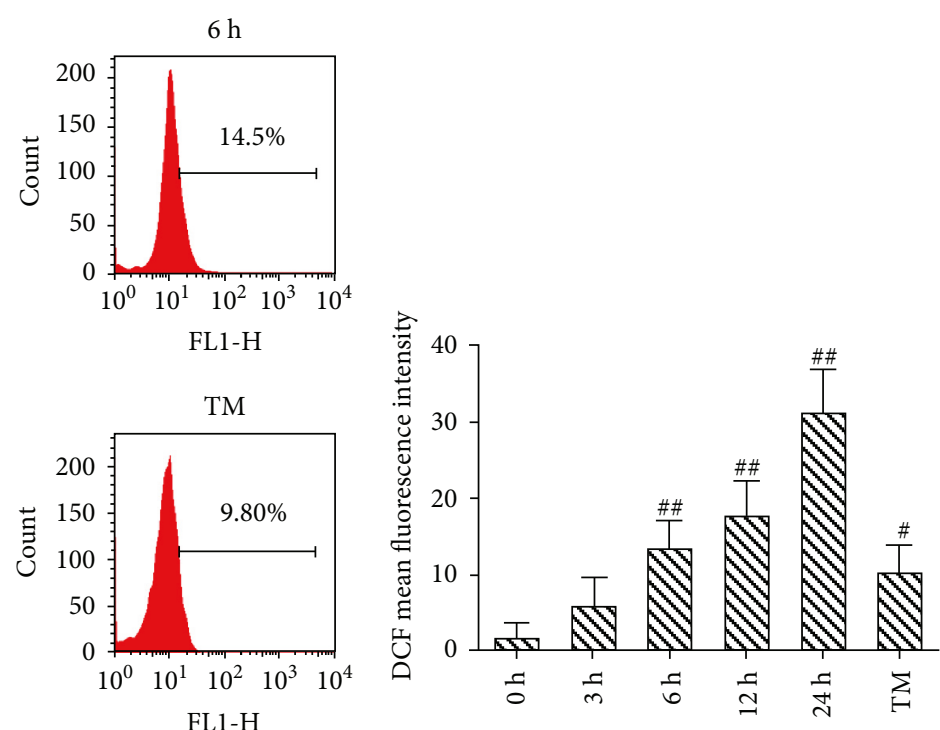

(e)

FIgUre 1: Continued. 

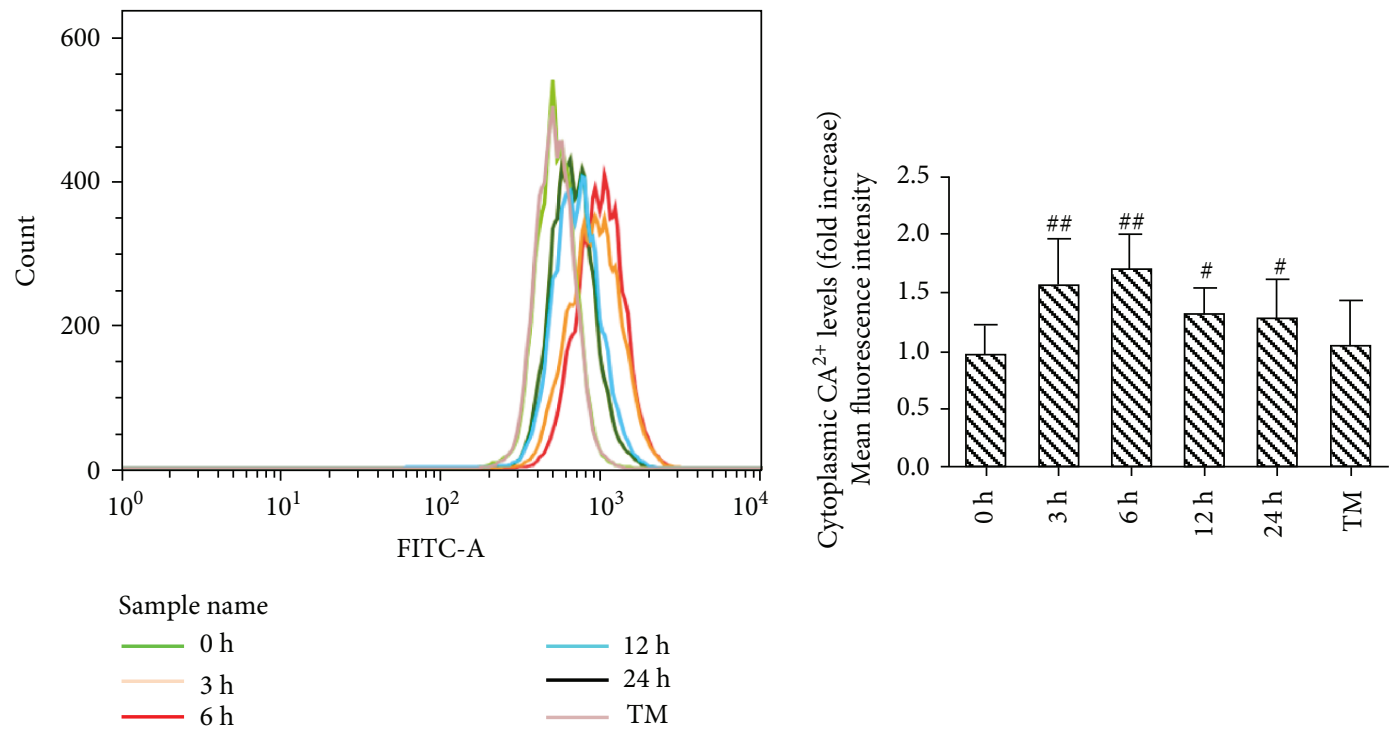

(f)

$(\mathrm{g})$

FIGURE 1: Earle's balanced salt solution (EBSS) increases NADPH oxidative activity and ROS generation in ARPE-19 cells in a timedependent manner. $(\mathrm{a}-\mathrm{c})$ A Western blot analysis was carried out to detect the expression levels of NOXs. (d, e) Intracellular ROS was determined by flow cytometry. (f, g) Earle's balanced salt solution (EBSS) increases cytoplasmic calcium in ARPE-19 cells. The intracellular calcium was detected with Fluo-3 AM. TM stands for tunicamycin. The data are presented as the means \pm SEM of three independent experiments. ${ }^{\#} p<0.05$ and ${ }^{\# \#} p<0.01$ compared to control $(0 \mathrm{~h})$.

derived ROS. Furthermore, the ROS level and nuclear calpain-2 induction might be crucial pathogenic elements for apoptosis of cardiomyocyte [28].

There is growing evidence showing that NOXs are important sources of ROS during ER stress [29]. The increase of cellular stress and oxidative stress can lead to ER stress by activating the process of unfolded protein response and $\mathrm{Ca}^{2+}$ disturbances [30, 31]. Numerous studies have revealed that oxidative stress and ER stress are associated with neuronal cell death signaling after ischemia injury [32]. Oxidative stress plays an essential role in protective cell autophagy $[33,34]$. ROS also leads to excessive autophagy and even apoptosis in cells [35]. It is known that apoptosis of ARPE-19 cells is the major cause of AMD-induced pathological changes [36]. In addition, a change in ROS balance is in charge of the execution of cell apoptosis $[37,38]$.

RPE cells are highly metabolically active cells that are located in the retina and play a vital role in maintaining normal visual function. Hence, RPE cells are vulnerable to oxidative stress $[7,39]$. In addition, sunlight is one of the causes for ROS production that can damage RPE cells $[40,41]$. The human cell line ARPE-19 has functional and structural characteristics similar to RPE cells. Therefore, we used an ARPE-19 cell line for our study.

In our previous study, we demonstrated that taurine inhibited starvation-triggered cell damage in ARPE-19 cells [24]. However, the detailed roles of NOX (one of the main ROS sources) in EBSS-induced cell injury remain unclear. We hypothesize that NOXs are the upstream regulators of calpains, ER stress, autophagy, and apoptosis. Furthermore, the protective effect of taurine is mediated by the reduction of NOX-derived ROS, leading to sequential suppression of calpain induction, ER stress, autophagy, and apoptosis. In this study, the potential roles of NOXs in EBSS-induced cell injury were studied in ARPE-19 cells.

\section{Material and Methods}

2.1. Cell Line and Cell Culture. ARPE-19 cells were procured from Shanghai GuanDao Biotech Co. Ltd. Cells were subcultured in Dulbecco's modified Eagle's medium (DMEM)/F-12 (Hyclone, Logan, UT, USA) containing 10\% FBS (Gibco, Grand Island, NY, USA), penicillin $(100 \mathrm{IU} / \mathrm{mL})$, and streptomycin $(100 \mu \mathrm{g} / \mathrm{mL})$. Cells were maintained at $37^{\circ} \mathrm{C}$ and $5 \% \mathrm{CO}_{2}$.

2.2. Transfection Experiments. Human NOX2, NOX4, p22phox, and NOX5 small interfering RNAs (siRNAs) were obtained from GenePharma (ShangHai, China). The siRNAs (two short sequences of siRNA specific for each one) were transfected into ARPE-19 cells using lipofectamine 2000 reagent (Invitrogen, Carlsbad, CA, USA) for $12 \mathrm{~h}$. Next, cells were incubated for another $48 \mathrm{~h}$ in normal culture conditions. Protein expression was detected using Western blot, and the better siRNAs were chosen for subsequent experiments. The scrambled (nontargeting) siRNAs were used as the negative control.

2.3. Western Blotting. The protocol of the Western blot analysis is described in detail elsewhere [24]. Antibodies used and their dilutions were the following: NOX1 (1:500, Cambridge, MA, USA, Abcam, ab55831), NOX2/gp91phox (1:500, ABclonal Biotech, Wuhan, China, A1636), NOX4 (1:1000, Abcam, ab133303), p22phox (1:1000, Abcam, ab191512), 

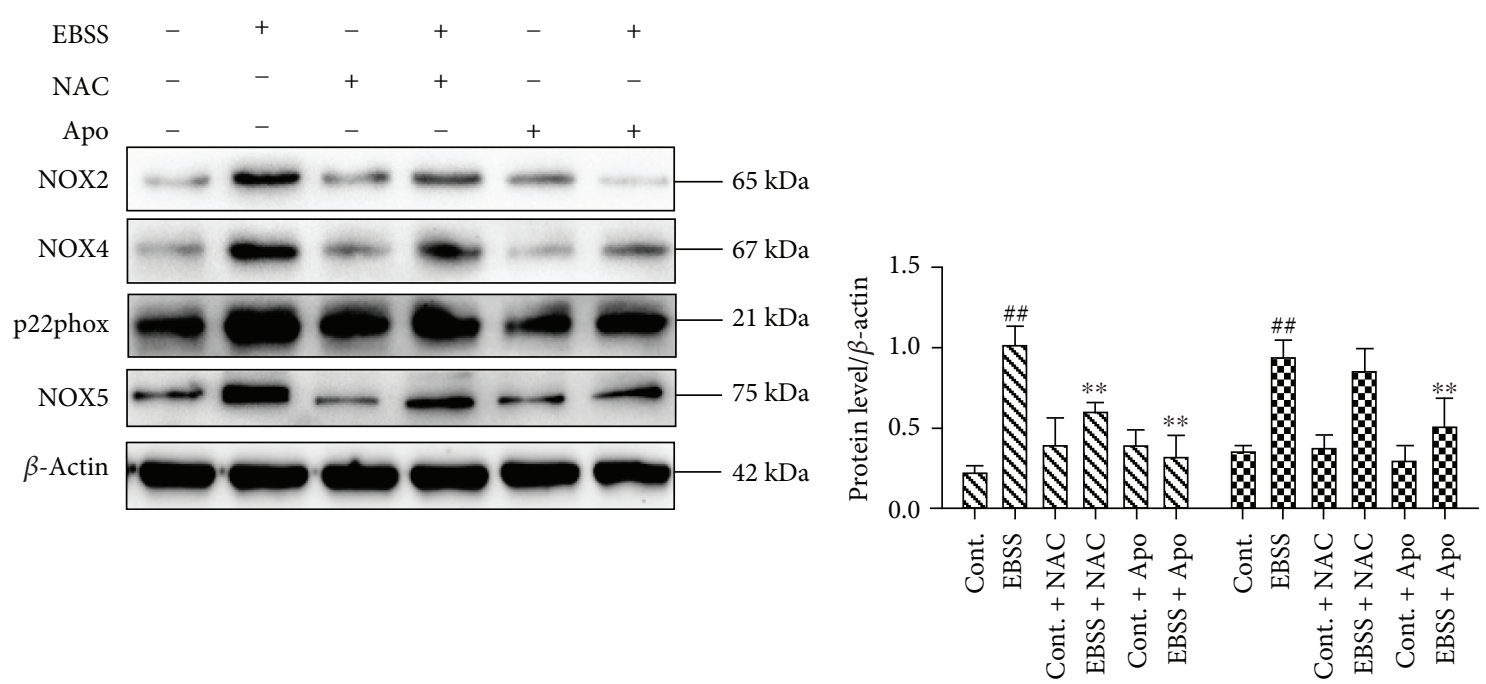

$\triangle 1$ NOX2

mo NOX4

(a)

(b)

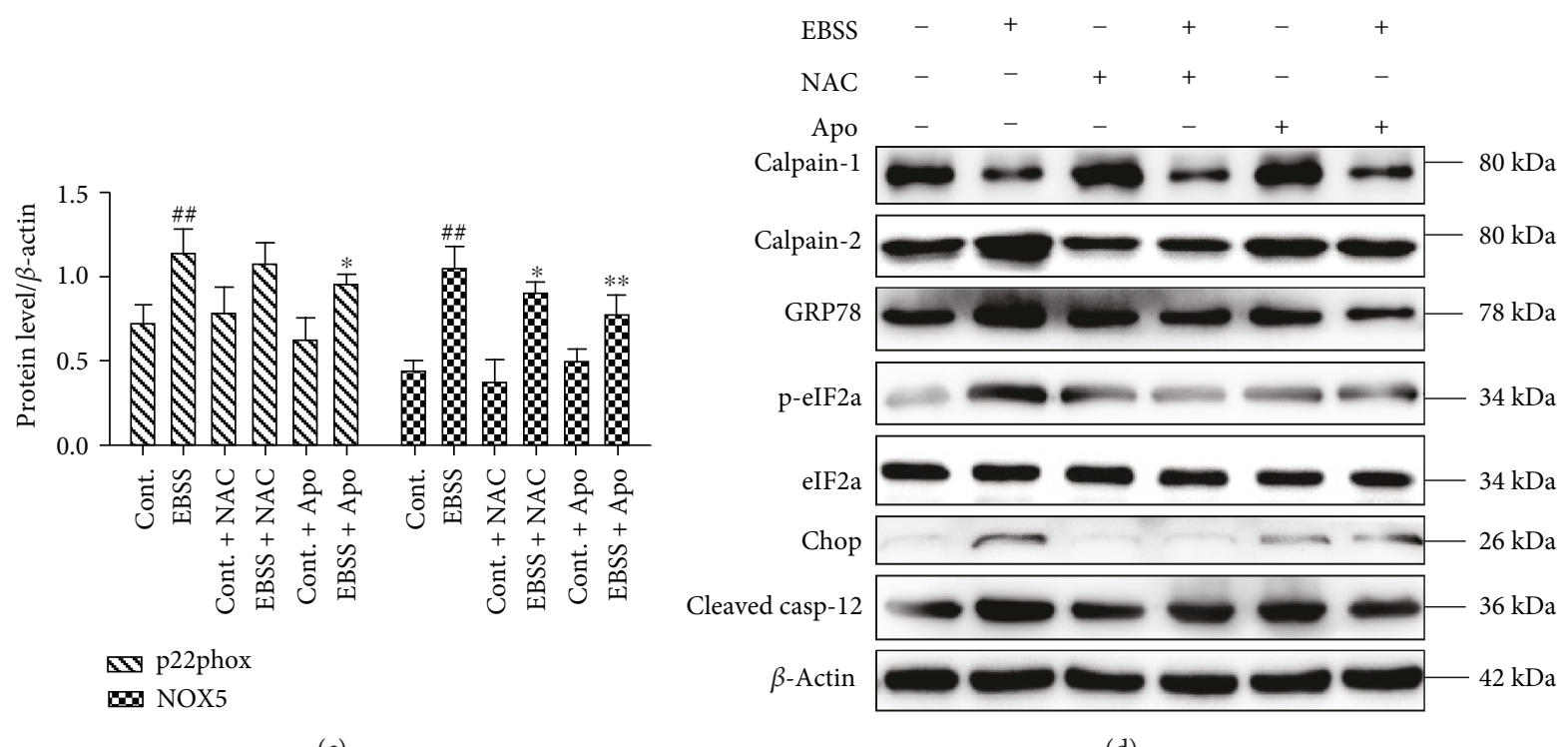

(c)

(d)

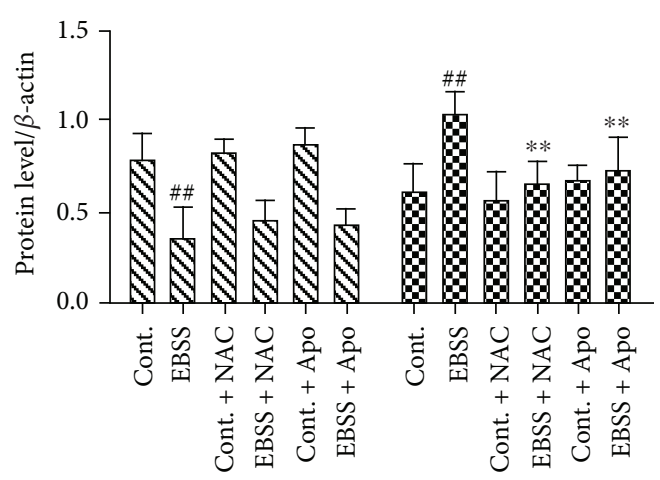

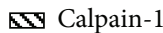
$\infty$ Calpain-2

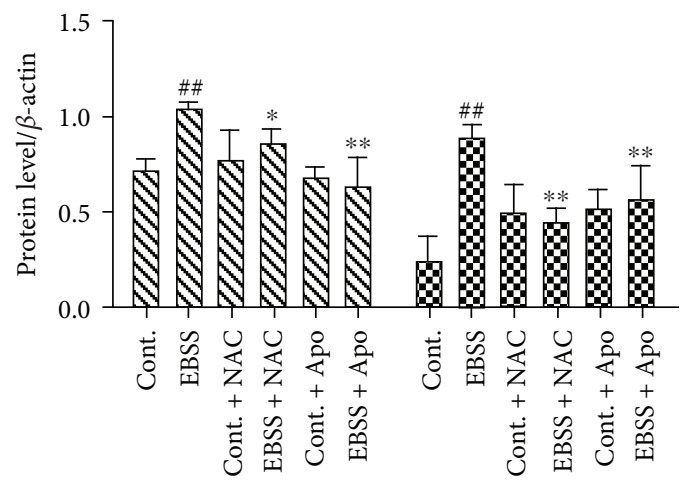

GN GR78

$\infty$ p-eIF2a

(e)

Figure 2: Continued. 


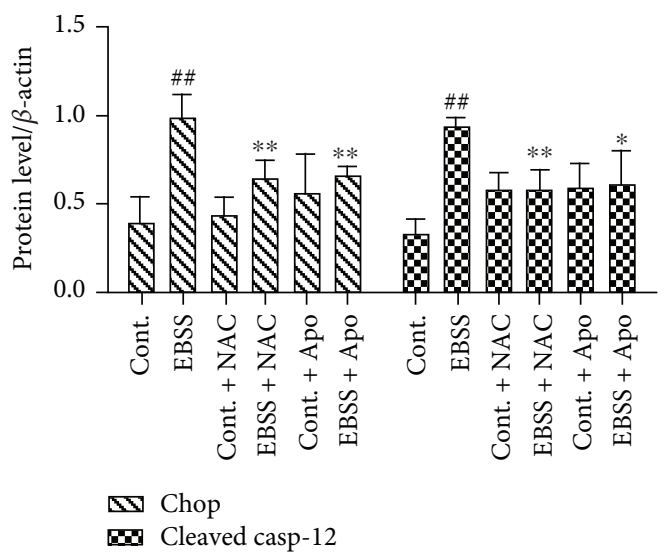

(g)

FIGURE 2: ROS scavenging and NOX suppression attenuate EBSS-induced loss of calcium homeostasis and ER stress in ARPE-19 cells. (a-c) The expression levels of NOX proteins were examined by Western blot. (d-g) The expression levels of calpains and the ER stress-related proteins were examined by Western blot. The data are presented as the means \pm SEM of three independent experiments. ${ }^{\# \#} p<0.01$ compared to the control group. ${ }^{*} p<0.05$ and ${ }^{* *} p<0.01$ compared to the EBSS group.

and NOX5 (1:500, ABclonal, A7136). Other antibodies are described in detail elsewhere [24].

2.4. Flow Cytometry. Cellular ROS levels were monitored using the fluorescent probe (DCFH-DA). Cells were seeded in 6-well plates overnight and treated with EBSS for $24 \mathrm{~h}$ with or without inhibitors/siRNA/taurine pretreatment. Next, cells were loaded with DCFH-DA $(10 \mu \mathrm{M})$ in PBS for $30 \mathrm{~min}$. Fluorescence was measured with a FACSCalibur flow cytometer (Becton, Dickinson and Company, San Jose, CA, USA). The protocol of the annexin V/PI staining is described in detail elsewhere [24]. The cytosolic-free calcium was detected using Fluo-3 AM. The protocol of the intracellular calcium detection is described in detail elsewhere [42].

2.5. Confocal Microscopy. Cells were cocultured with DCFHDA (10 $\mu \mathrm{M}$, Beyotime Biotech, ShangHai, China) for $30 \mathrm{~min}$. Nuclei were stained with Hoechst for $20 \mathrm{~min}$. The protocol of image analysis is described in detail elsewhere [24].

2.6. Statistics. Data was expressed as the mean \pm standard error of the mean (SEM). Statistics were analyzed by SPSS 21.0 statistics program (SPSS Inc., Chicago, IL, USA). One-way ANOVA and Dunnett's posttest were used to determine the statistical significance. A $p$ value of less than $0.05(p<0.05)$ was considered significant.

\section{Results}

3.1. Effects of EBSS Treatment on NOX Expression and ROS Generation. To evaluate the role of EBSS treatment in the activities of NOXs and ROS generation, the cells were cultured in EBSS for 0, 3, 6, 12, and 24 hours (h) or with tunicamycin $(50 \mu \mathrm{M})$ as a positive control for $6 \mathrm{~h}$. The expression of NOX proteins was examined by Western blot. The results show that the protein expression of NOX2, NOX4, p22phox, and NOX5 was dramatically increased from $6 \mathrm{~h}$ until $24 \mathrm{~h}$ after EBSS treatment. However, the expression of NOX1 was unchanged (Figures 1(a)-1(c)). The generation of ROS was markedly elevated between $6 \mathrm{~h}$ and $24 \mathrm{~h}$ after EBSS treatment (Figures 1(d) and 1(e)). Our results suggest that EBSS induces the activities of NOXs and ROS production. In addition, levels of NOXs were fully activated by $6 \mathrm{~h}$ posttreatment and subsequently this time point was chosen for our experiments. ER stress, autophagy, and apoptosis also could be induced at 6 and $12 \mathrm{~h}$, respectively (data available online with this article; doi: 10.3390/ijms18102146, pages 4-5). We observed that the fluorescence intensity of the peaks shifted immediately after $3 \mathrm{~h}$ until $24 \mathrm{~h}$ post-EBSS treatment. Furthermore, EBSS induced the loss of calcium homeostasis in ARPE-19 cells (Figures 1(f) and 1(g)).

3.2. ROS Scavenging and NOX Suppression Attenuated EBSS-Induced Loss of Calcium Homeostasis and ER Stress. To investigate the possible roles of NOXs and ROS in EBSS-induced loss of calcium homeostasis and ER stress in ARPE-19 cells, the cells were pretreated for $2 \mathrm{~h}$ with the ROS scavenger compound, N-acetyl-cysteine (NAC, $10 \mathrm{mM}$ ), or the NOX inhibitor, apocynin (Apo, $1 \mathrm{mM})$, and then cultured with EBSS ( 6 or $12 \mathrm{~h}$ ). We found that pretreatment with NAC or Apo strongly and significantly reduced the expression of NOX2, NOX4, p22phox, and NOX5 (Figures 2(a)-2(c)), suggesting that the activity of NOXs and the accumulation of ROS were inhibited by the two inhibitors. To assess the relationship between NOXs, ER stress, and calpains in ARPE-19 cells, calpains and ER stress-related proteins were examined by Western blot. The results show that the expression of calpain-2 and ER stress-related proteins was decreased. However, induction of calpain-1 was unchanged (Figures $2(\mathrm{~d})-2(\mathrm{~g})$ ). Our findings suggest that the induction of NOXs can promote loss of calcium homeostasis and ER stress. Furthermore, NOXs may be critical upstream regulators of calcium homeostasis and ER stress.

3.3. NOX Suppression and ROS Scavenging Attenuated EBSS-Induced Autophagy, Apoptosis, and ROS Generation. 


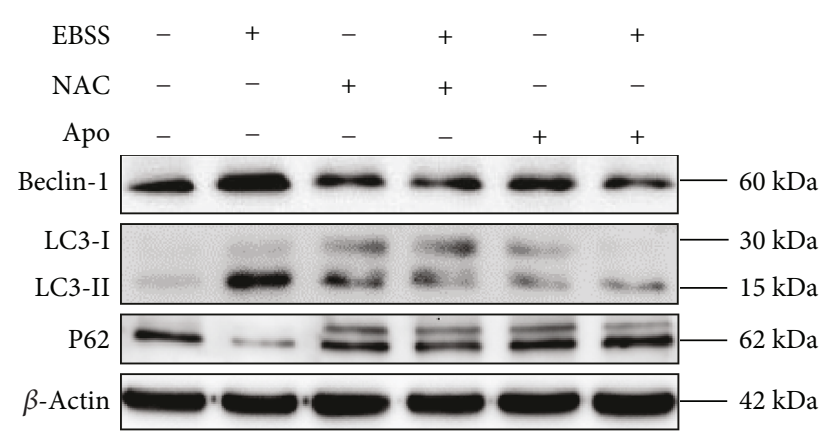

(a)

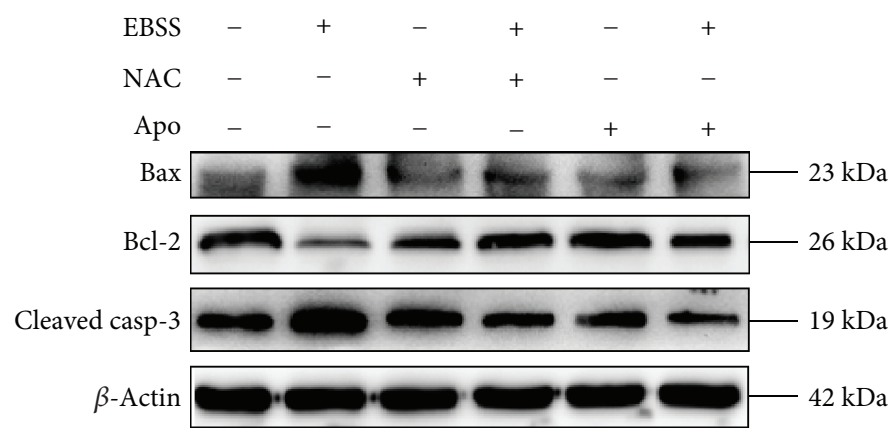

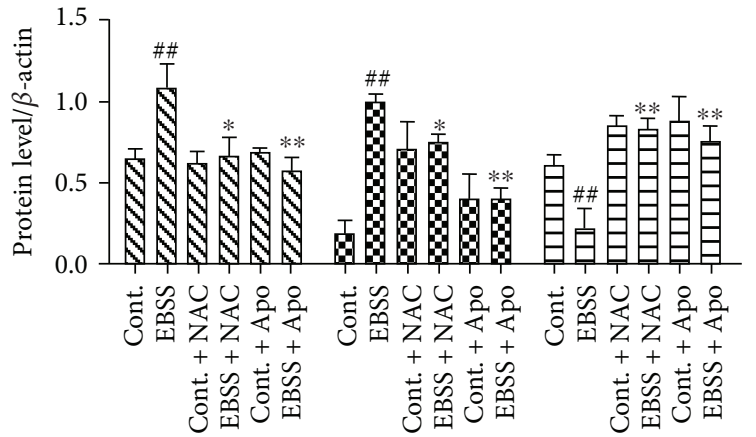

$\Delta$ Beclin-1

LC3 II

曰 P62

(b)

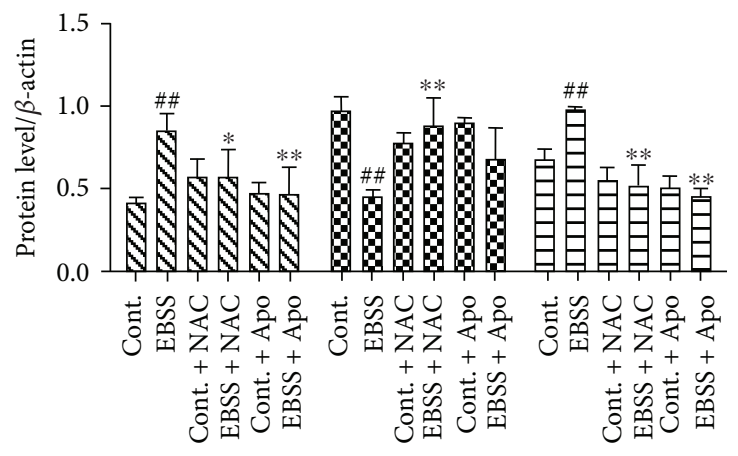

$\triangle \mathrm{Bax}$

$6 \mathrm{Bcl}-2$

$\boxminus$ Cleaved casp-3

(d)

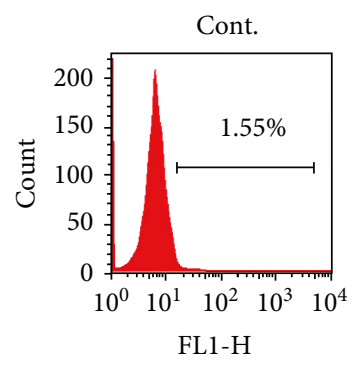

(c)
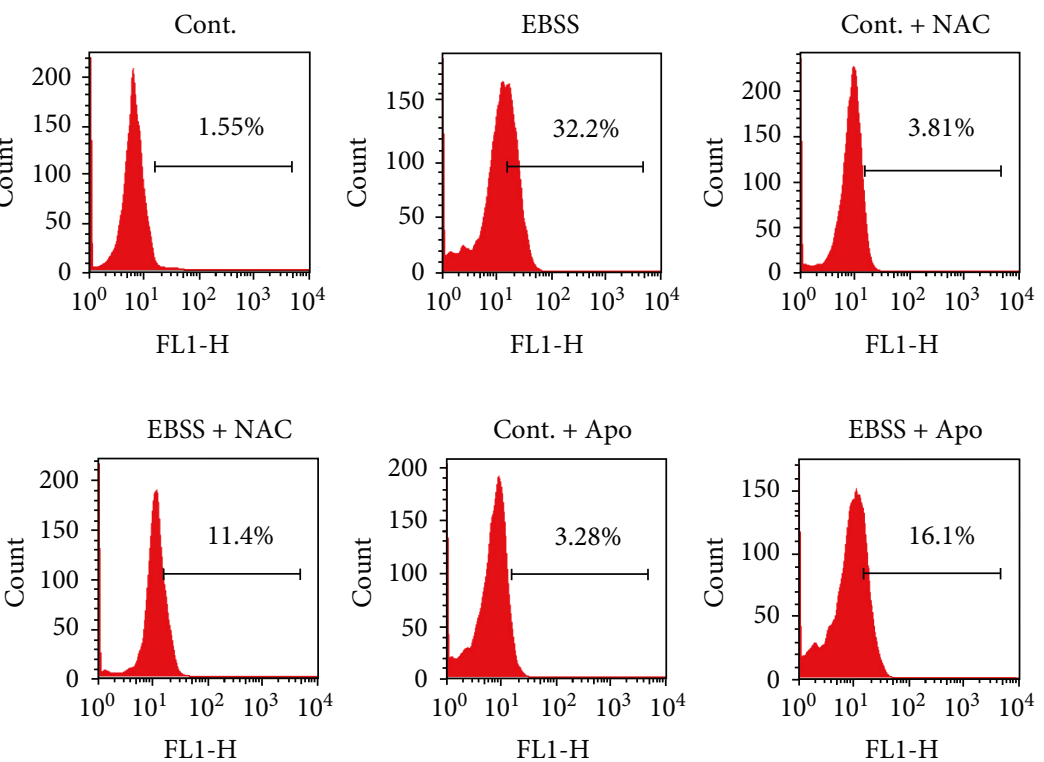

(e)

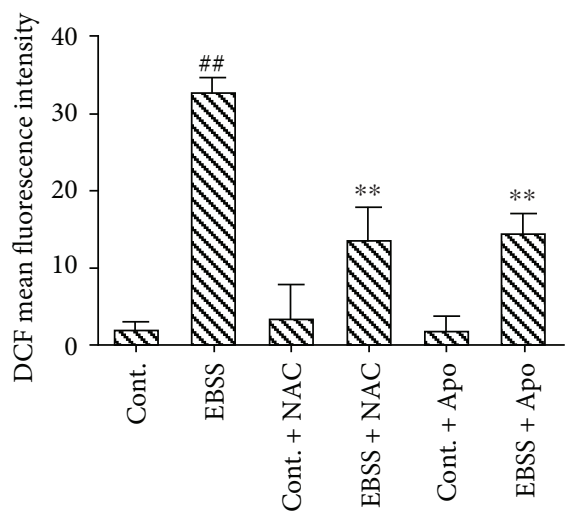

(f)

FIgURE 3: ROS scavenging and NOX suppression attenuate EBSS-induced autophagy, apoptosis, and ROS generation. (a-d) The expression of autophagy-related proteins and apoptosis-related proteins was detected by Western blot. (e, f) Intracellular ROS was evaluated by flow cytometry. The data are presented as the means \pm SEM of three independent experiments. \#\# $p<0.01$ compared to the control group. ${ }^{*} p<0.05$ and ${ }^{* *} p<0.01$ compared to the EBSS group. 

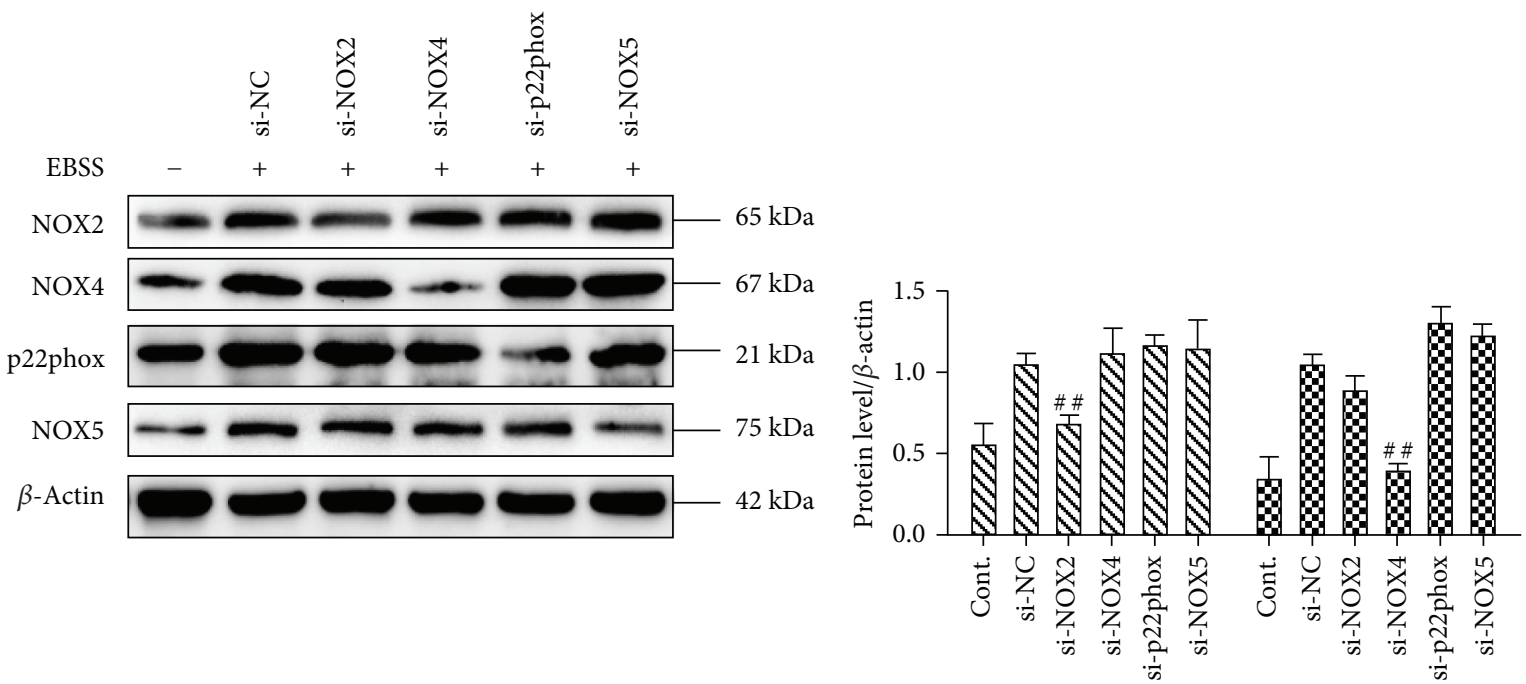

$\mathbb{N O N} 2$

$\boldsymbol{\infty}$ NOX4

(b)

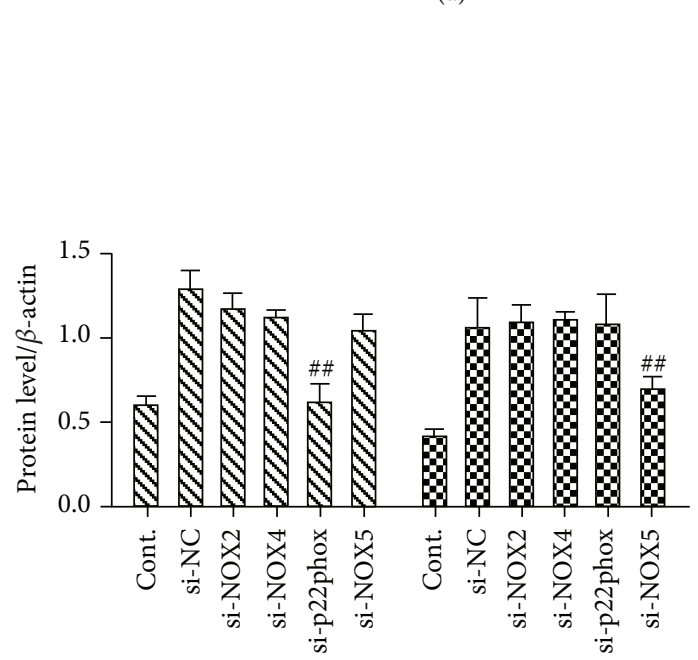

प1v p22phox

- 0 NOX5

(c)

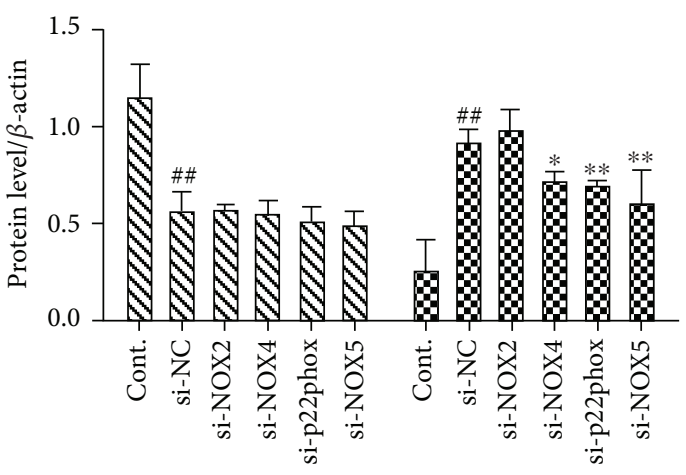

W. Calpain-1

Walpain-2

(e)

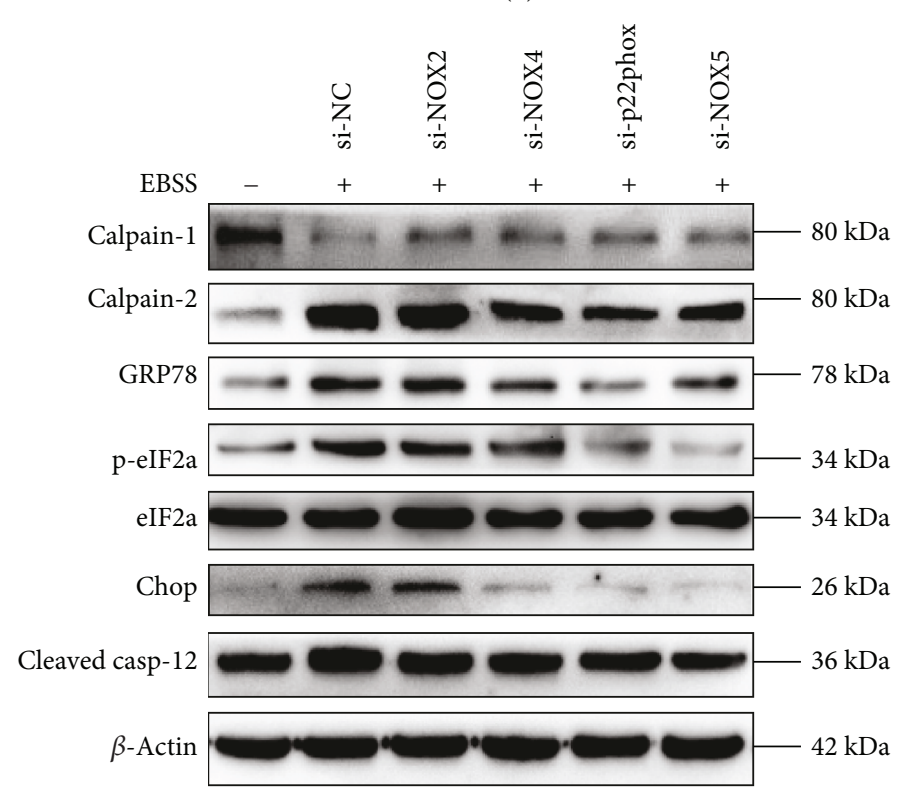

(d)

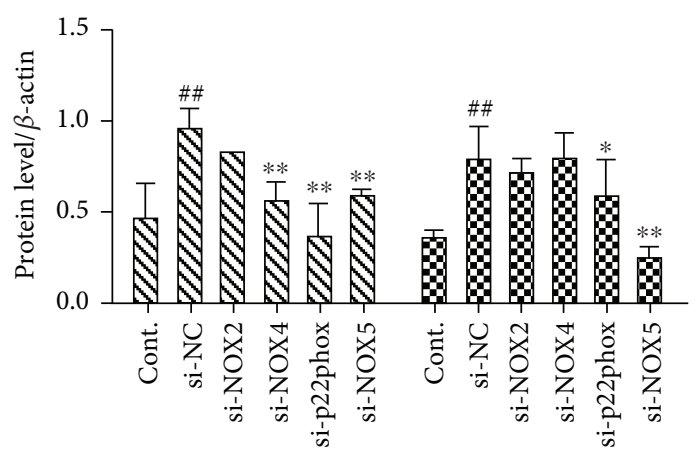

GRP78

seIF2a

Figure 4: Continued. 


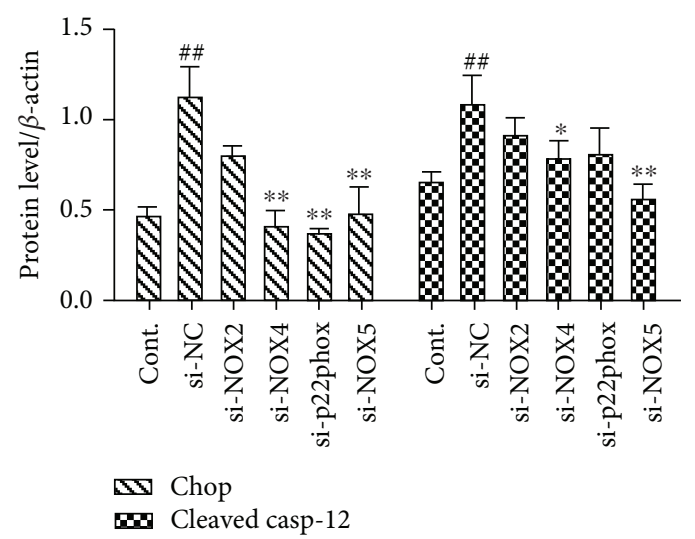

(g)

FIgURE 4: NOX4, p22phox, and NOX5 modulate loss of calcium homeostasis and ER stress in ARPE-19 cells. (a-c) Silencing of NOX2/NOX4/ p22phox/NOX5 decreases its protein level. (d-g) The activities of ER stress and calpain-2 were suppressed by siRNA knockdown of NOX4, p22phox, and NOX5. The data are presented as the means \pm SEM of three independent experiments. \#\# $p<0.01$ compared to the control group. ${ }^{*} p<0.05$ and ${ }^{* *} p<0.01$ compared with the EBSS group.

To further explore the possible roles of NOXs and ROS in EBSS-induced autophagy and apoptosis in ARPE-19 cells, we examined the expression of autophagy-related proteins and mitochondrial apoptotic proteins by Western blot. Our results suggest that both inhibitors, NAC and Apo, caused a significant decrease in the expression of beclin1, LC3, Bax, and cleaved caspase-3. Meanwhile, levels of P62 and Bcl-2 were noticeably increased (Figures 3(a)$3(d)$ ), indicating that the NOXs were related to the induction of autophagy and apoptosis. To evaluate the role of ROS, intracellular ROS was first evaluated by flow cytometry. The results show that pretreatment with NAC or Apo reverses the accumulation of ROS (Figures 3(e) and 3(f)). Our findings show that the suppression of NOX oxidases or ROS generation could decrease autophagy and apoptosis. Moreover, NOXs may be vital upstream regulators of ER stress, autophagy, and apoptosis.

3.4. NOX4, p22phox, and NOX5 Played Crucial Roles in EBSS-Induced Loss of Calcium Homeostasis and ER Stress. To test the involvement of NOXs in EBSS-induced calpain production and ER stress, we used specific interference RNA (siRNA) species leading to a marked decrease of NOX protein levels (Figures 4(a)-4(c)). To demonstrate the effect of NOXs on ER stress and calpain production, calpains and the ER stress-related proteins were examined by Western blot. The results show that NOX4, p22phox, and NOX5 silencing resulted in lower levels of calpain-2 and ER stress-related proteins. However, induction of calpain-1 was unaffected (Figures 4(d)-4(g)). Our findings indicate that silencing NOX4, p22phox, and NOX5 can prevent calpain induction and ER stress. Furthermore, NOX4, p22phox, and NOX5 may be essential upstream regulators of calcium homeostasis and ER stress.

3.5. NOX4, p22phox, and NOX5 Play Key Roles in EBSSInduced Autophagy, Apoptosis, and ROS Generation. We next evaluated the effects of NOX2, NOX4, p22phox, and NOX 5 on autophagy, apoptosis, and ROS production. The autophagy-related proteins and apoptosis-related proteins were examined by Western blot. The results show that NOX4, p22phox, and NOX5 silencing downregulated the expression of beclin-1, LC3, Bax, and cleaved caspase-3. Meanwhile, the levels of P62 and Bcl-2 were increased (Figures 5(a)-5(d)). Moreover, the cell apoptosis rate was evaluated by flow cytometry, and our data show that apoptosis is inhibited with the reduction of NOX4, p22phox, and NOX5 expression (Figures 5(e) and 5(f)). The main sources of ROS are NOX enzymes, and our data suggest that the downregulation of NOX4, p22phox, and NOX5 is associated with significantly reduced production of ROS (Figures 5(g) and 5(h)). Our findings indicate that silencing of NOX4, p22phox, and NOX5 can reduce autophagy, apoptosis, and ROS production. Furthermore, NOX4, p22phox, and NOX5 may be essential upstream regulators of autophagy and apoptosis.

3.6. Effects of Taurine on NOX Expression and ROS Generation Induced by EBSS. We also investigated the effects of taurine $(30 \mathrm{mM})$ on NOXs and ROS. Our results suggest that the expression of NOX2, NOX4, p22phox, and NOX5 in the EBSS group was increased compared with the control group. The level of proteins was decreased after intervention with taurine. The expression of NOX1 levels was not influenced in models (EBSS treatment) and taurine + EBSS groups (Figures 6(a)-6(c)). Oxidative stress was evaluated by detecting ROS with flow cytometry. Intracellular ROS was decreased by taurine treatment (Figures 6(d) and 6(e)). Moreover, ROS accumulation was also determined with immunofluorescence. High fluorescence intensity was presented in the EBSS group. But it was weak in the control and EBSS + taurine groups (Figures 6(f) and 6(g)). These data suggest that taurine inhibited the activities of NOX enzymes and the accumulation of ROS. Therefore, taurine attenuates ER stress, autophagy, and apoptosis in ARPE-19 cells via suppression of the NADPH oxidase-derived reactive oxygen species-mediated calpain induction pathway (data 

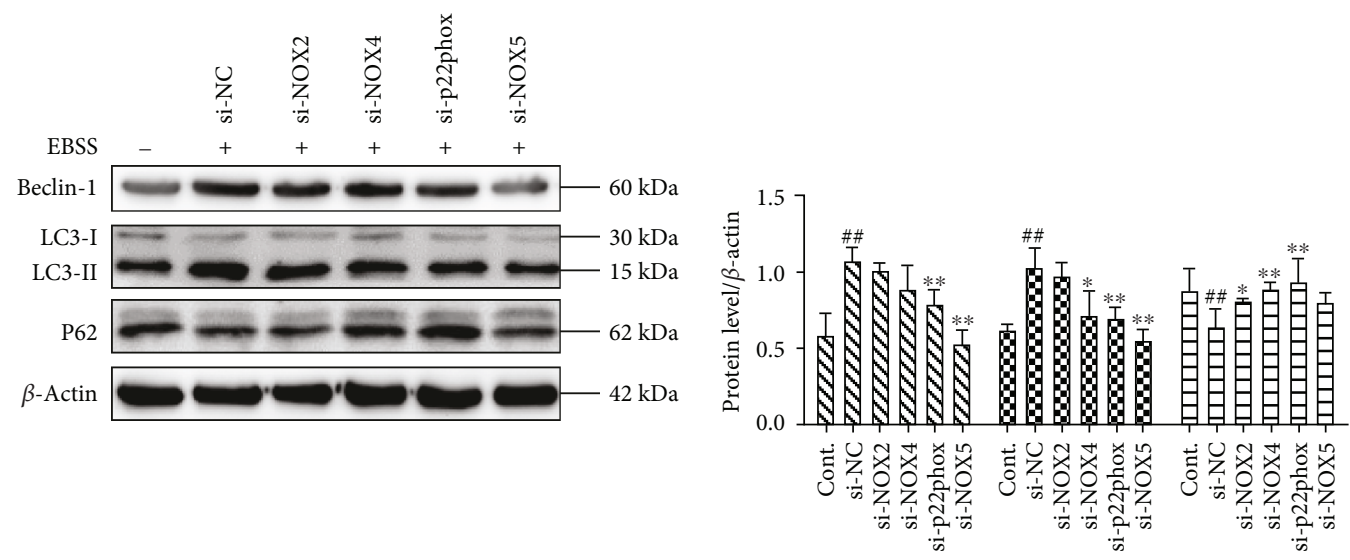

(\$) Beclin-1

LC LC3 II

$\boxminus \mathrm{P} 62$

(a)

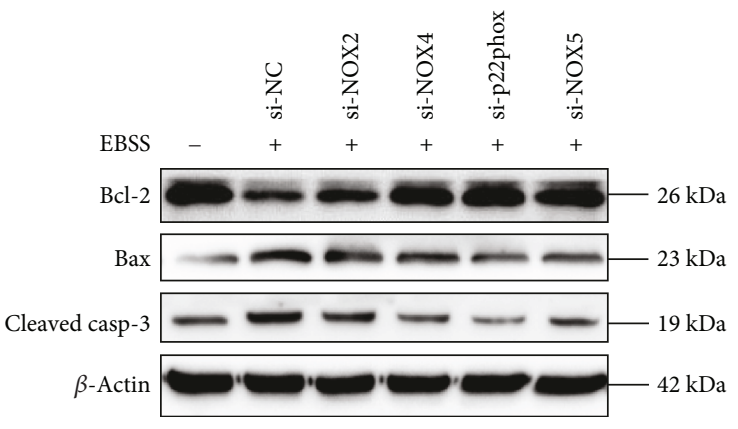

(b)

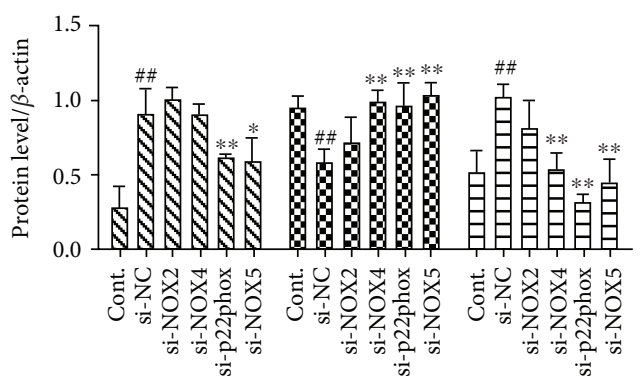

$\Delta \mathrm{Bax}$

Bis $\mathrm{Bcl}-2$

$\boxminus$ Cleaveland casp-3

(d)
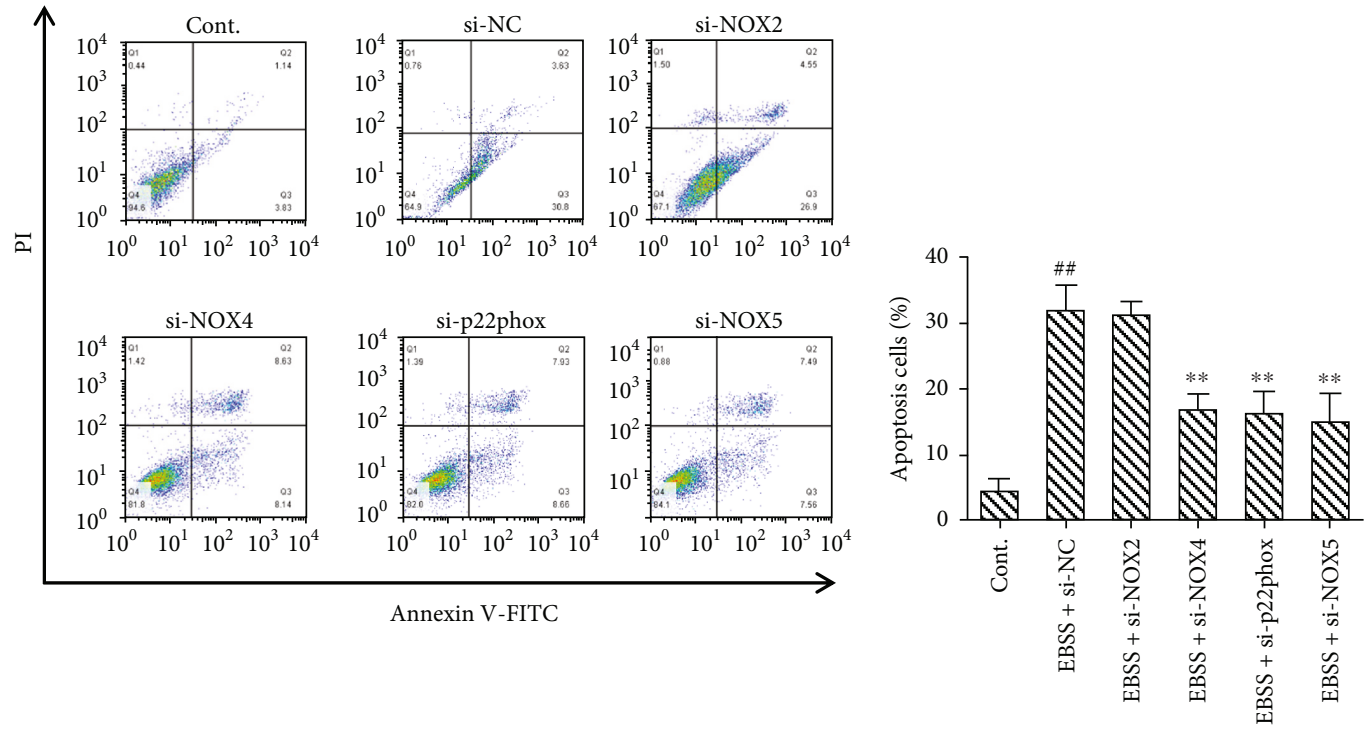

(e)

(f)

Figure 5: Continued. 

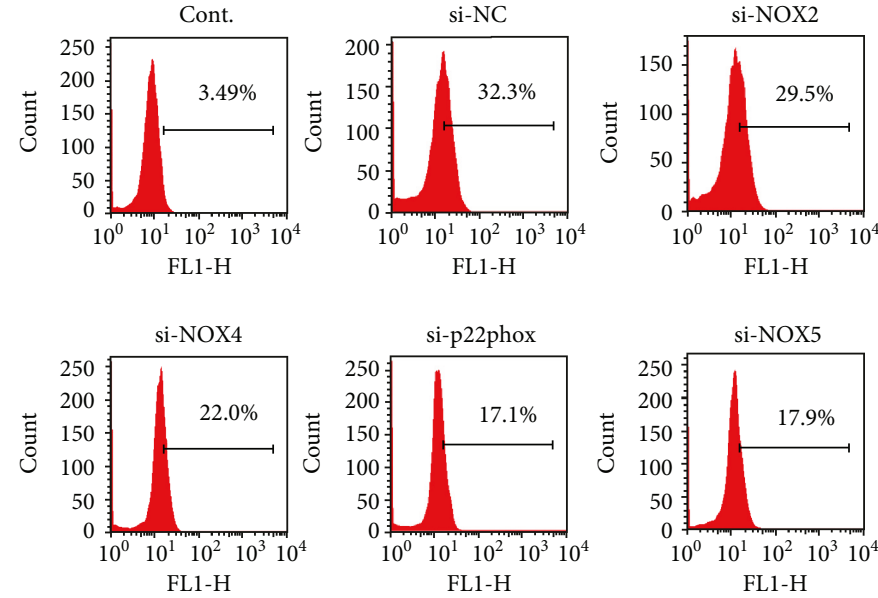

(g)

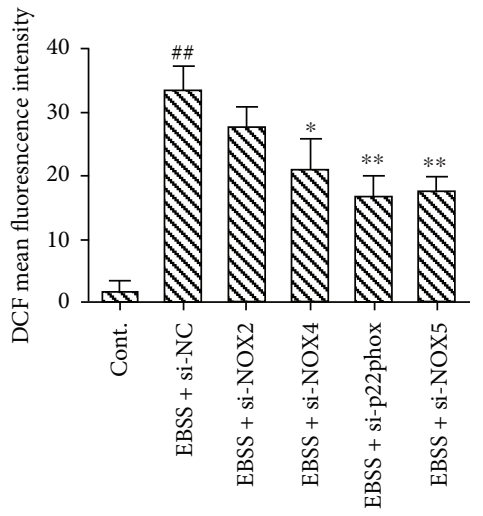

(h)

FIgURE 5: NOX4, p22phox, and NOX5 modulate autophagy and apoptosis in ARPE-19 cells. (a-d) NOX4, p22phox, or NOX5 silencing inhibits autophagy and apoptosis. (e, f) siRNA knockdown of NOX4, p22phox, or NOX5 decreases cell apoptosis. (g, h) siRNA knockdown of NOX4, p22phox, or NOX5 decreases ROS production. Intracellular ROS was evaluated by flow cytometry. The data are presented as the means \pm SEM of three independent experiments. ${ }^{\# \#} p<0.01$ compared to the control group. ${ }^{*} p<0.05$ and ${ }^{* *} p<0.01$ compared to the EBSS group.

available online with this article; doi:10.3390/ijms18102146, pages $8-9)$.

\section{Discussion}

Our study demonstrates the involvement of NOX-derived ROS in EBSS-induced RPE metabolic disorders. NOXs are upstream regulators of calpain-2, ER stress, autophagy, and apoptosis. Furthermore, NOXs are involved in calpain-2, ER stress, autophagy, and apoptosis regulation by NOX4, p22phox, and NOX5 but not by NOX1 or NOX2. Taurine alleviated cell injuries through regulation of NOX levels (Figure 7).

During metabolic stress (starvation), the balance of ROS level changes. NOXs are activated and result in excessive production of ROS (termed oxidative stress) (Figure 1). Furthermore, an excessive level of ROS interferes with lipids and proteins leading to functional and structural changes of target molecules $[43,44]$. NOX1/NADPH oxidase plays a key role in endotoxin-induced cardiomyocyte apoptosis [19]. NOX1 does not influence vascular endothelial growth factor (VEGF) activation in HG-induced cell injuries [16]. NOX2 and NOX4 play physiological roles in homocysteineinduced endothelial cell apoptosis [45]. NOX5 and p22phox are involved in human monocyte differentiation into dendritic cells [3]. We demonstrate that NOX4 and p22phox play key roles in ARPE-19 cell injuries (Figure 1). NOX5 is expressed in cells of the cardiovascular system and in the retina [46]. However, NOX5 is absent in rodents and this may explain the lack of data on its expression and function in the retina in this model. Previous research indicated that NOX5 was predominantly expressed in RPE cells [15]. Our results show that NOX5 is a key factor of EBSS-induced cell damage (Figure 1).

The interaction of ROS and calcium signaling can be considered bidirectional; ROS can regulate cellular calcium signaling, while calcium signaling is crucial for ROS generation [25, 47, 48]. Increasing evidence suggests that this crosstalk plays a key role in many pathological conditions, including colorectal cancer, cardiomyocyte apoptosis, neuronal damage, and also renal cell injury [28, 49-51]. We revealed that suppression of NOX-mediated ROS production through the use of the NOX inhibitor (apocynin) or ROS scavenger (NAC) inhibited calpain-2 induction (Figure 2). During cellular stress, NOX-mediated ROS generation was observed. ROS levels correlate with cell survival. The decrease in ROS production also reduces the expression of NOX, which forms a feed-forward loop [52-55]. NOX is activated due to its notable ability to produce ROS. The ER is a major site of calcium storage [25]. The release of $\mathrm{Ca}^{2+}$ also leads to ROS accumulation [56, 57]. Overproduction of ROS activated ER-based calcium channels by triggering signaling molecules upstream of calpain-2 [58-60]. Induction of calpain-2 further mediates the subsequent ER-mitochondrial crosstalk $[1,28,50]$. ROS not only acts as an activator but can also be regulated by autophagy through macroautophagosomes $[34,61]$. Bcl-2 is an integral membrane protein, and it can form homodimers to protect cells from apoptosis. In contrast, Bax is a proapoptotic gene and can cause mitochondria to release cytochrome $\mathrm{C}$. Cytochrome $\mathrm{C}$ leads to caspase 3 activation which is an important mitochondrial apoptotic marker $[62,63]$. We investigated the ROS-calcium crosstalk in the $\mathrm{ER}$, autophagosome, and their influence on apoptosis in the mitochondria. Our results indicate that suppression of NOX oxidases or ROS generation could decrease calpain- 2 induction, ER stress, autophagy, and apoptosis (Figures 2 and 3).

To elucidate the functional significance of NOXs, we examined ROS generation, ER stress, autophagy, and apoptosis in which NOX2/NOX4/p22phox/NOX5 was knocked down with siRNA. Although NOX4/p22phox/NOX5 was not completely knocked down, induction of NADPH oxidase was inhibited. The exact reasons for this are complex and 


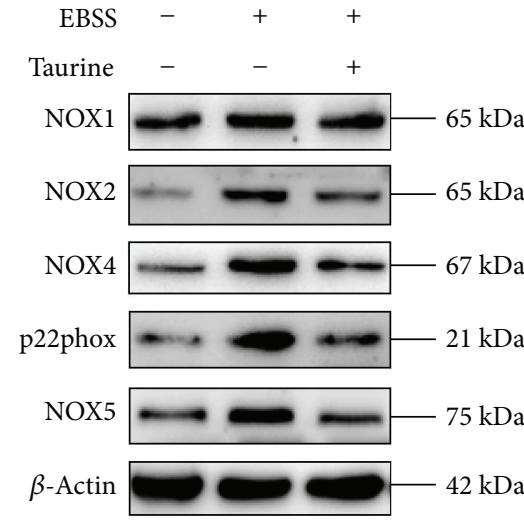

(a)

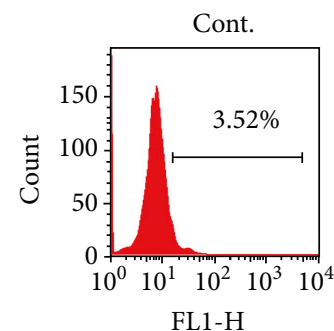

FL1-H

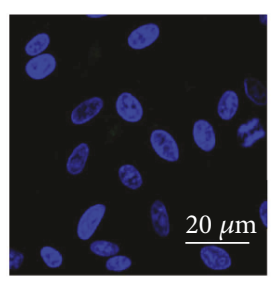

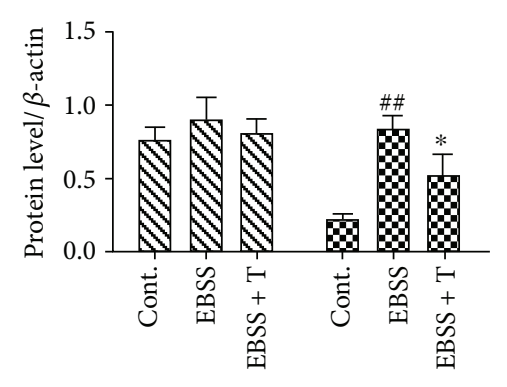

$\triangle$ NOX1

NOX2

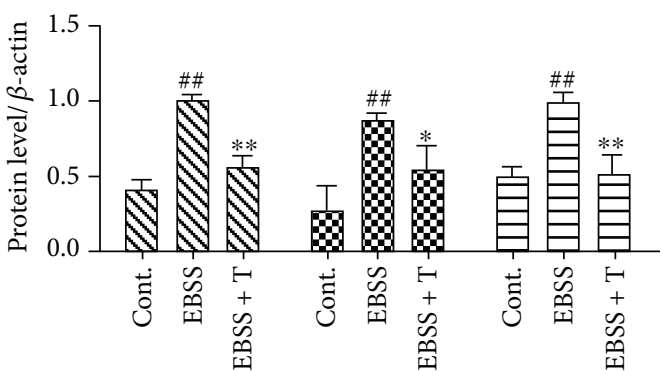

$\triangle \mathrm{NOX} 4$

p22phox

$\boxminus$ NOX5 (b)

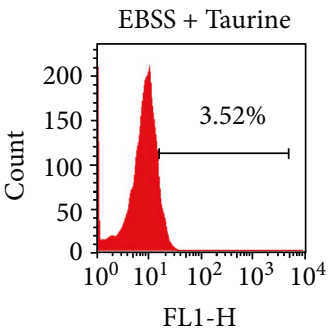

(d)

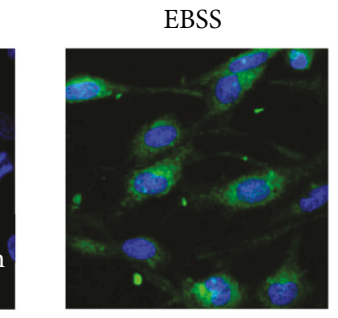

(f)
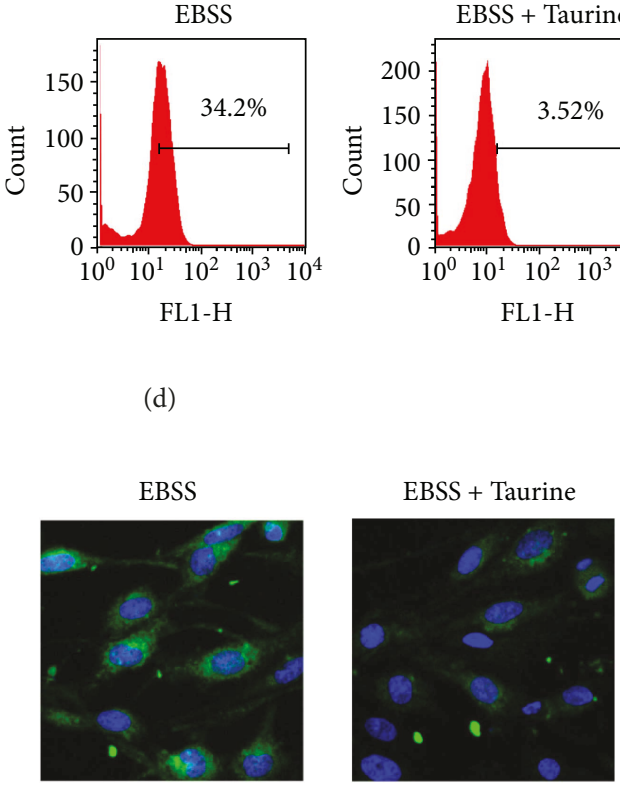

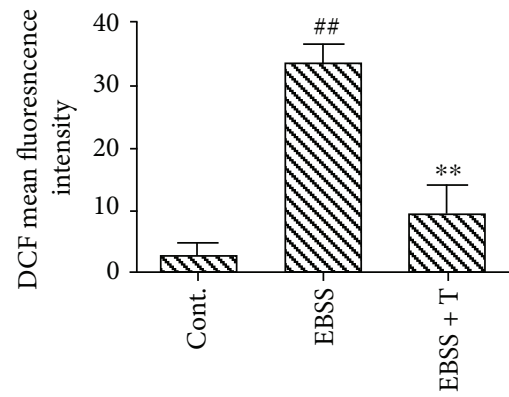

(e)

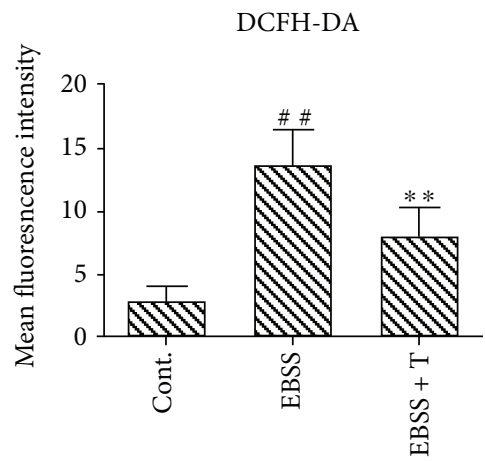

(g)

FIgURE 6: Effects of taurine on NOX expression and ROS generation induced by EBSS. (a-c) Western blot analysis was carried out to determine the expression of NOX proteins in ARPE-19 cells. (d, e) Intracellular ROS was evaluated by flow cytometry. (f, g) Confocal images of ROS labelled with DCFH-DA (green) and nuclear stained with DAPI (blue). Scale bar $=20 \mu \mathrm{m}$. The data are presented as the means \pm SEM of three to five independent experiments. ${ }^{\# \#} p<0.01$ compared to the control group. ${ }^{*} p<0.05$ and ${ }^{* *} p<0.01$ compared to the EBSS group. T: taurine.

not well-understood but relate to the fact that NOX4/ p22phox/NOX5 downregulation influences other NADPH oxidase subunits that may have an impact on the induction of oxidases $[45,64]$. It is also possible that NOX4/
p22phox/NOX5 might interact with other NOX isoforms, which, in the context of NOX4/p22phox/NOX5 downregulation, inhibits NOX-associated NOX induction. Such considerations require further examinations. In NOX4/p22phox/ 


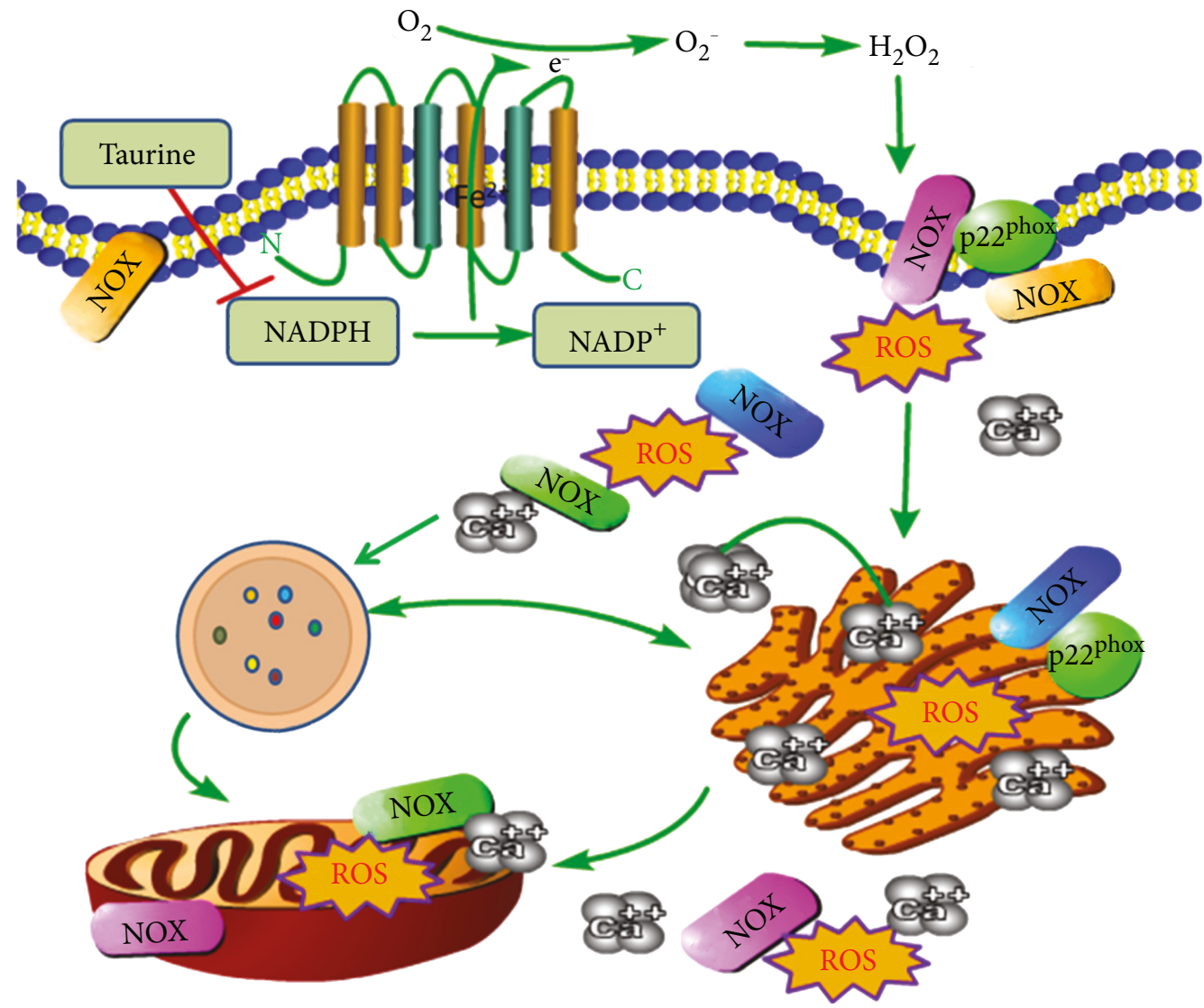

FIGURE 7: Schematic diagram of the effects of taurine in ARPE-19 cells. The protective effect of taurine is regulated by the reduction of intracellular ROS from NOXs, leading, in turn, to sequential suppression of calpain induction, ER stress, autophagy, and apoptosis. NADPH oxidases (NOXs) are transmembrane proteins that are localized either in intracellular granules and vesicles or on the cell surface membranes.

NOX5-downregulated cells, but not NOX2-downregulated cells, ROS generation, ER stress, autophagy, and apoptosis were significantly reduced, indicating the importance of NOX4/p22phox/NOX5 in redox signaling by EBSS. Moreover, these processes are $\mathrm{Ca}^{2+}$ sensitive, because calpain-2 induction was also inhibited. Hence, NOX influence generation of ROS also regulates ER stress, autophagy, and apoptosis. We showed that NOX4, p22phox, and NOX5 are critical for damage and cell death of ARPE-19 cells. In addition, we found for the first time that NOX5 induction is required for calpain2 induction, ER stress, autophagy, and apoptosis in ARPE-19 cells (Figures 4 and 5).

The antioxidation of taurine may prevent disease progression [65]. The antioxidant properties are limited by the sulfonic group through neutralizing ROS production $[41,66]$. In a previous study, we demonstrated that taurine inhibited starvation-triggered cell damage. In our study, our results suggest that taurine inhibited the activation of NOXs and ROS generation (Figure 6).

In conclusion, our data demonstrate that ROS generated via the NADPH oxidase system is the major contributing factor in RPE dysfunction induced by EBSS. NOX silencing or suppression partly protects cells from cell damage. NOX4, p22phox, and NOX5 play key roles in the production of ROS, ER stress, autophagy, and apoptosis. Our observations support the hypothesis that oxidative stress is the main causative for cell injury, and taurine attenuates ER stress, autophagy, and apoptosis in ARPE-19 cells via a suppression of the NADPH oxidase-derived reactive oxygen species-mediated calpain induction pathway. Our data provides novel insights into ocular diseases and mechanisms of taurine.

\section{Data Availability}

Previously reported research data were used to support this study and are available at doi: 10.3390/ijms18102146. These prior studies (and datasets) are cited at relevant places within the text as references. The data used to support the findings of this study are available from the corresponding author, Zhou Zhang, upon reasonable request.

\section{Conflicts of Interest}

The authors declare no conflict of interest.

\section{Authors' Contributions}

Yuanyuan Zhang conceived and designed the experiments. Yuanyuan Zhang and Shu Ren performed the experiments. Zhou Zhang, Yuanyuan Zhang, Yanting Gu, and Jiahong 
Wang analyzed the data. Zheng Liu contributed reagents, materials, and analysis tools. Yuanyuan Zhang wrote the paper.

\section{Acknowledgments}

This research was funded by the National Natural Science Foundation of China (Grant no. 31270883) to Zhou Zhang.

\section{References}

[1] M. L. Sheu, C. C. Shen, Y. S. Chen, and C. K. Chiang, "Ochratoxin A induces ER stress and apoptosis in mesangial cells via a NADPH oxidase-derived reactive oxygen speciesmediated calpain activation pathway," Oncotarget, vol. 8, no. 12, pp. 19376-19388, 2017.

[2] G. Ren, W. Luo, W. Sun et al., "Psoralidin induced reactive oxygen species (ROS)-dependent DNA damage and protective autophagy mediated by NOX4 in breast cancer cells," Phytomedicine, vol. 23, no. 9, pp. 939-947, 2016.

[3] V. Marzaioli, M. Hurtado-Nedelec, C. Pintard et al., "NOX5 and p22phox are 2 novel regulators of human monocytic differentiation into dendritic cells," Blood, vol. 130, no. 15, pp. 1734-1745, 2017.

[4] A. Kowluru and R. A. Kowluru, "Phagocyte-like NADPH oxidase [Nox2] in cellular dysfunction in models of glucolipotoxicity and diabetes," Biochemical Pharmacology, vol. 88, no. 3, pp. 275-283, 2014.

[5] J. L. Wilkinson-Berka, I. Rana, R. Armani, and A. Agrotis, "Reactive oxygen species, Nox and angiotensin II in angiogenesis: implications for retinopathy," Clinical Science, vol. 124, no. 10, pp. 597-615, 2013.

[6] S. Huang, P. Huang, Z. Lin et al., "Hydrogen sulfide supplement attenuates the apoptosis of retinal ganglion cells in experimental glaucoma," Experimental Eye Research, vol. 168, pp. 33-48, 2018.

[7] Y. Li, X. Liu, T. Zhou et al., "Inhibition of APE1/Ref-1 redox activity rescues human retinal pigment epithelial cells from oxidative stress and reduces choroidal neovascularization," Redox Biology, vol. 2, pp. 485-494, 2014.

[8] M. Sugai, A. Ohta, Y. Ogata et al., "Asymmetric dimethylarginine (ADMA) in the aqueous humor of diabetic patients," Endocrine Journal, vol. 54, no. 2, pp. 303-309, 2007.

[9] G. Mohammad, K. Alam, M. I. Nawaz, M. M. Siddiquei, A. Mousa, and A. M. Abu El-Asrar, "Mutual enhancement between high-mobility group box-1 and NADPH oxidasederived reactive oxygen species mediates diabetes-induced upregulation of retinal apoptotic markers," Journal of Physiology and Biochemistry, vol. 71, no. 3, pp. 359-372, 2015.

[10] J. Li, J. J. Wang, Q. Yu, K. Chen, K. Mahadev, and S. X. Zhang, "Inhibition of reactive oxygen species by lovastatin downregulates vascular endothelial growth factor expression and ameliorates blood-retinal barrier breakdown in $d b / d b$ mice: role of NADPH oxidase 4," Diabetes, vol. 59, no. 6, pp. 15281538, 2010.

[11] M. Sedeek, A. C. Montezano, R. L. Hebert et al., "Oxidative stress, Nox isoforms and complications of diabetes-potential targets for novel therapies," Journal of Cardiovascular Translational Research, vol. 5, no. 4, pp. 509-518, 2012.
[12] E. C. Vaquero, M. Edderkaoui, S. J. Pandol, I. Gukovsky, and A. S. Gukovskaya, "Reactive oxygen species produced by $\mathrm{NAD}(\mathrm{P}) \mathrm{H}$ oxidase inhibit apoptosis in pancreatic cancer cells," Journal of Biological Chemistry, vol. 279, no. 33, pp. 34643-34654, 2004.

[13] Y. Wang, L. Zhong, X. Liu, and Y. Z. Zhu, "ZYZ-772 prevents cardiomyocyte injury by suppressing Nox4-derived ROS production and apoptosis," Molecules, vol. 22, no. 2, 2017.

[14] J. E. Leadsham, G. Sanders, S. Giannaki et al., "Loss of cytochrome $\mathrm{c}$ oxidase promotes RAS-dependent ROS production from the ER resident NADPH oxidase, Ynolp, in yeast," Cell Metabolism, vol. 18, no. 2, pp. 279-286, 2013.

[15] J. C. Jha, A. M. D. Watson, G. Mathew, L. C. de Vos, and K. Jandeleit-Dahm, "The emerging role of NADPH oxidase NOX5 in vascular disease," Clinical Science, vol. 131, no. 10, pp. 981-990, 2017.

[16] R. Haque, P. M. Iuvone, L. He et al., "Prorenin receptor (PRR)mediated NADPH oxidase (Nox) signaling regulates VEGF synthesis under hyperglycemic condition in ARPE-19 cells," Journal of Receptor and Signal Transduction, vol. 37, no. 6, pp. 560-568, 2017.

[17] Y. Qiu, L. Tao, C. Lei et al., "Downregulating p22phox ameliorates inflammatory response in angiotensin II-induced oxidative stress by regulating MAPK and NF- $\kappa \mathrm{B}$ pathways in ARPE-19 cells," Scientific Reports, vol. 5, no. 1, article 14362, 2015.

[18] D. Deliyanti and J. L. Wilkinson-Berka, "Inhibition of NOX1/4 with GKT137831: a potential novel treatment to attenuate neuroglial cell inflammation in the retina," Journal of Neuroinflammation, vol. 12, no. 1, p. 136, 2015.

[19] K. Matsuno, K. Iwata, M. Matsumoto et al., "NOX1/NADPH oxidase is involved in endotoxin-induced cardiomyocyte apoptosis," Free Radical Biology and Medicine, vol. 53, no. 9, pp. 1718-1728, 2012.

[20] Y. Y. Qin, M. Li, X. Feng et al., "Combined NADPH and the NOX inhibitor apocynin provides greater anti-inflammatory and neuroprotective effects in a mouse model of stroke," Free radical biology and medicine, vol. 104, pp. 333-345, 2017.

[21] K. K. Prior, M. S. Leisegang, I. Josipovic et al., "CRISPR/Cas9mediated knockout of p22phox leads to loss of Nox1 and Nox4, but not Nox5 activity," Redox biology, vol. 9, pp. 287295, 2016

[22] F. Chen, Y. Wang, S. Barman, and D. J. Fulton, "Enzymatic regulation and functional relevance of NOX5," Current Pharmaceutical Design, vol. 21, no. 41, pp. 5999-6008, 2015.

[23] S. Carnesecchi, A. L. Rougemont, J. H. Doroshow et al., "The NADPH oxidase NOX5 protects against apoptosis in ALKpositive anaplastic large-cell lymphoma cell lines," Free Radical Biology and Medicine, vol. 84, pp. 22-29, 2015.

[24] Y. Zhang, S. Ren, Y. Liu, K. Gao, Z. Liu, and Z. Zhang, "Inhibition of starvation-triggered endoplasmic reticulum stress, autophagy, and apoptosis in ARPE-19 cells by taurine through modulating the expression of calpain-1 and calpain-2," International Journal of Molecular Sciences, vol. 18, no. 10, 2017.

[25] A. Gorlach, K. Bertram, S. Hudecova, and O. Krizanova, "Calcium and ROS: a mutual interplay," Redox biology, vol. 6, pp. 260-271, 2015.

[26] S. Limbu, T. M. Hoang-Trong, B. L. Prosser, W. J. Lederer, and M. S. Jafri, "Modeling local X-ROS and calcium signaling in the heart," Biophysical Journal, vol. 109, no. 10, pp. $2037-$ 2050, 2015. 
[27] S. Javadov, "The calcium-ROS-pH triangle and mitochondrial permeability transition: challenges to mimic cardiac ischemiareperfusion," Frontiers in Physiology, vol. 6, p. 83, 2015.

[28] H. Chang, J. J. Sheng, L. Zhang et al., "ROS-induced nuclear translocation of calpain-2 facilitates cardiomyocyte apoptosis in tail-suspended rats," Journal of Cellular Biochemistry, vol. 116, no. 10, pp. 2258-2269, 2015.

[29] F. R. Laurindo, T. L. Araujo, and T. B. Abrahao, "Nox NADPH oxidases and the endoplasmic reticulum," Antioxidants \& Redox Signaling, vol. 20, no. 17, pp. 2755-2775, 2014.

[30] H. Y. Lee, H. J. Chae, S. Y. Park, and J. H. Kim, "Porcine placenta hydrolysates enhance osteoblast differentiation through their antioxidant activity and effects on ER stress," BMC Complementary and Alternative Medicine, vol. 16, no. 1, p. 291, 2016.

[31] C. X. Santos, A. A. Nabeebaccus, A. M. Shah, L. L. Camargo, S. V. Filho, and L. R. Lopes, "Endoplasmic reticulum stress and Nox-mediated reactive oxygen species signaling in the peripheral vasculature: potential role in hypertension," Antioxidants \& Redox Signaling, vol. 20, no. 1, pp. 121-134, 2014.

[32] S. Hong, J. Kwon, D. W. Kim, H. J. Lee, D. Lee, and W. Mar, "Mulberrofuran G protects ischemic injury-induced cell death via inhibition of NOX4-mediated ROS generation and ER stress," Phytotherapy Research, vol. 31, no. 2, pp. 321-329, 2017.

[33] E. J. Song, S. J. Lee, H. S. Lim et al., "Vibrio vulnificus VvhA induces autophagy-related cell death through the lipid raftdependent c-Src/NOX signaling pathway," Scientific Reports, vol. 6 , no. 1, article 27080, 2016.

[34] S. Sciarretta, P. Zhai, D. Shao et al., "Activation of NADPH oxidase 4 in the endoplasmic reticulum promotes cardiomyocyte autophagy and survival during energy stress through the protein kinase RNA-activated-like endoplasmic reticulum kinase/eukaryotic initiation factor $2 \alpha$ /activating transcription factor 4 pathway," Circulation research, vol. 113, no. 11, pp. 1253-1264, 2013.

[35] X. Wen, J. Wu, F. Wang, B. Liu, C. Huang, and Y. Wei, "Deconvoluting the role of reactive oxygen species and autophagy in human diseases," Free Radical Biology and Medicine, vol. 65, pp. 402-410, 2013.

[36] L. Palanza, R. Nuzzi, M. Repici, and A. Vercelli, "Ganglion cell apoptosis and increased number of NADPH-d-positive neurones in the rodent retina in an experimental model of glaucoma," Acta Ophthalmologica Scandinavica, vol. 80, pp. 47-48, 2002.

[37] J. Dong, K. K. Sulik, and S. Y. Chen, "The role of NOX enzymes in ethanol-induced oxidative stress and apoptosis in mouse embryos," Toxicology letters, vol. 193, no. 1, pp. 94$100,2010$.

[38] P. Sancho, E. Bertran, L. Caja, I. Carmona-Cuenca, M. M. Murillo, and I. Fabregat, "The inhibition of the epidermal growth factor (EGF) pathway enhances TGF- $\beta$-induced apoptosis in rat hepatoma cells through inducing oxidative stress coincident with a change in the expression pattern of the NADPH oxidases (NOX) isoforms," Biochimica et Biophysica Acta (BBA) - Molecular Cell Research, vol. 1793, no. 2, pp. 253-263, 2009.

[39] S. H. Kim, H. Kim, H. J. Ku et al., "Oxalomalate reduces expression and secretion of vascular endothelial growth factor in the retinal pigment epithelium and inhibits angiogenesis: implications for age-related macular degeneration," Redox biology, vol. 10, pp. 211-220, 2016.
[40] X. Zhu, K. Wang, K. Zhang, F. Zhou, and L. Zhu, "Induction of oxidative and nitrosative stresses in human retinal pigment epithelial cells by all-trans-retinal," Experimental cell research, vol. 348, no. 1, pp. 87-94, 2016.

[41] Y. Li, J. M. Arnold, M. Pampillo, A. V. Babwah, and T. Peng, "Taurine prevents cardiomyocyte death by inhibiting NADPH oxidase-mediated calpain activation," Free radical biology and medicine, vol. 46, no. 1, pp. 51-61, 2009.

[42] S. Lv, Q. Y. Xu, E. C. Sun, J. K. Zhang, and D. L. Wu, "Dissection and integration of the autophagy signaling network initiated by bluetongue virus infection: crucial candidates ERK1/2, Akt and AMPK," Scientific Reports, vol. 6, no. 1, article 23130, 2016.

[43] U. Weyemi, C. E. Redon, T. Aziz et al., "Inactivation of NADPH oxidases NOX4 and NOX5 protects human primary fibroblasts from ionizing radiation-induced DNA damage," Radiation Research, vol. 183, no. 3, pp. 262-270, 2015.

[44] S. Hu, X. Yang, and J. He, "NADPH oxidase-induced macrophage autophagy mediated by reactive oxygen species in Aspergillus fumigatus infection," Xi bao yu fen zi mian yi xue $z a$ zhi = Chinese journal of cellular and molecular immunology, vol. 31, no. 2, pp. 190-193, 2015.

[45] J. A. Sipkens, N. Hahn, C. S. van den Brand et al., "Homocysteine-induced apoptosis in endothelial cells coincides with nuclear NOX2 and peri-nuclear NOX4 activity," Cell biochemistry and biophysics, vol. 67, no. 2, pp. 341-352, 2013.

[46] T. J. Guzik, W. Chen, M. C. Gongora et al., "Calciumdependent NOX5 nicotinamide adenine dinucleotide phosphate oxidase contributes to vascular oxidative stress in human coronary artery disease," Journal of the American College of Cardiology, vol. 52, no. 22, pp. 1803-1809, 2008.

[47] C. Mazars, P. Thuleau, O. Lamotte, and S. Bourque, "Crosstalk between ROS and calcium in regulation of nuclear activities," Molecular Plant, vol. 3, no. 4, pp. 706-718, 2010.

[48] A. Di, D. Mehta, and A. B. Malik, "ROS-activated calcium signaling mechanisms regulating endothelial barrier function," Cell Calcium, vol. 60, no. 3, pp. 163-171, 2016.

[49] M. Chocry, L. Leloup, and H. Kovacic, "Reversion of resistance to oxaliplatin by inhibition of p38 MAPK in colorectal cancer cell lines: involvement of the calpain / Nox1 pathway," Oncotarget, vol. 8, no. 61, pp. 103710-103730, 2017.

[50] B. Paramo, T. Montiel, D. R. Hernandez-Espinosa, M. RiveraMartinez, J. Moran, and L. Massieu, "Calpain activation induced by glucose deprivation is mediated by oxidative stress and contributes to neuronal damage," The international journal of biochemistry \& cell biology, vol. 45, no. 11, pp. 25962604, 2013.

[51] L. Yu, X. Gan, X. Liu, and R. An, "Calcium oxalate crystals induces tight junction disruption in distal renal tubular epithelial cells by activating ROS/Akt/p38 MAPK signaling pathway," Renal Failure, vol. 39, no. 1, pp. 440-451, 2017.

[52] D. Hernandez-Saavedra, L. Sanders, M. J. Perez et al., "RTP801 amplifies nicotinamide adenine dinucleotide phosphate oxidase-4-dependent oxidative stress induced by cigarette smoke," American Journal of Respiratory Cell and Molecular Biology, vol. 56, no. 1, pp. 62-73, 2017.

[53] C. Wilson, E. Munoz-Palma, D. R. Henriquez et al., "A feed-forward mechanism involving the NOX complex and RyR-mediated $\mathrm{Ca}^{2+}$ release during axonal specification," Journal of Neuroscience, vol. 36, no. 43, pp. 11107-11119, 2016. 
[54] Y. Cai, L. Yang, G. Hu et al., "Regulation of morphine-induced synaptic alterations: role of oxidative stress, ER stress, and autophagy," Journal of Cell Biology, vol. 215, no. 2, pp. 245258, 2016.

[55] G. L. Semenza and N. R. Prabhakar, "Neural regulation of hypoxia-inducible factors and redox state drives the pathogenesis of hypertension in a rodent model of sleep apnea," Journal of Applied Physiology, vol. 119, no. 10, pp. 1152-1156, 2015.

[56] R. K. Srivastava, C. Li, A. Ahmad et al., "ATF4 regulates arsenic trioxide-mediated NADPH oxidase, ER-mitochondrial crosstalk and apoptosis," Archives of Biochemistry and Biophysics, vol. 609, pp. 39-50, 2016.

[57] Y. S. Kim, S. J. Birge, L. V. Avioli, and R. Miller, "Cell densitydependent vitamin $\mathrm{D}$ effects on calcium accumulation in rat osteogenic sarcoma cells (ROS 17/2)," Calcified Tissue International, vol. 41, no. 4, pp. 218-222, 1987.

[58] M. S. Sharaf, D. Stevens, and C. Kamunde, "Zinc and calcium alter the relationship between mitochondrial respiration, ROS and membrane potential in rainbow trout (Oncorhynchus mykiss) liver mitochondria," Aquatic Toxicology, vol. 189, pp. 170-183, 2017.

[59] L. Minasyan, P. G. Sreekumar, D. R. Hinton, and R. Kannan, "Protective mechanisms of the mitochondrial-derived peptide Humanin in oxidative and endoplasmic reticulum stress in RPE cells," Oxidative Medicine and Cellular Longevity, vol. 2017, Article ID 1675230, 11 pages, 2017.

[60] U. Siegmund, J. Heller, J. A. van Kan, and P. Tudzynski, “The NADPH oxidase complexes in Botrytis cinerea: evidence for a close association with the ER and the tetraspanin Pls1," PLoS One, vol. 8, no. 2, article e55879, 2013.

[61] J. Huang, V. Canadien, G. Y. Lam et al., "Activation of antibacterial autophagy by NADPH oxidases," Proceedings of the National Academy of Sciences of the United States of America, vol. 106, no. 15, pp. 6226-6231, 2009.

[62] A. Beberok, D. Wrzesniok, J. Rok, Z. Rzepka, M. Respondek, and E. Buszman, "Ciprofloxacin triggers the apoptosis of human triple-negative breast cancer MDA-MB-231 cells via the $\mathrm{p} 53 / \mathrm{Bax} / \mathrm{Bcl}-2$ signaling pathway," International journal of oncology, vol. 52, no. 5, pp. 1727-1737, 2018.

[63] P. F. Hu, W. P. Chen, J. P. Bao, and L. D. Wu, "Paeoniflorin inhibits IL- $1 \beta$-induced chondrocyte apoptosis by regulating the Bax/Bcl-2/caspase-3 signaling pathway," Molecular Medicine Reports, vol. 17, no. 4, pp. 6194-6200, 2018.

[64] R. Accetta, S. Damiano, A. Morano et al., "Reactive oxygen species derived from NOX3 and NOX5 drive differentiation of human oligodendrocytes," Frontiers in cellular neuroscience, vol. 10, 2016.

[65] C. Bucolo, A. Fidilio, C. B. M. Platania, F. Geraci, F. Lazzara, and F. Drago, "Antioxidant and osmoprotecting activity of taurine in dry eye models," Journal of Ocular Pharmacology and Therapeutics, vol. 34, no. 1-2, pp. 188-194, 2017.

[66] N. Froger, L. Moutsimilli, L. Cadetti et al., "Taurine: the comeback of a neutraceutical in the prevention of retinal degenerations," Progress in Retinal and Eye Research, vol. 41, pp. 44-63, 2014. 


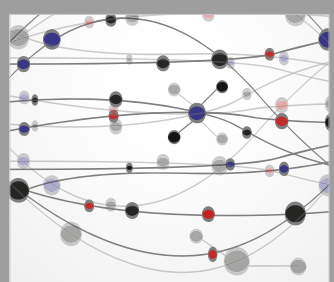

The Scientific World Journal
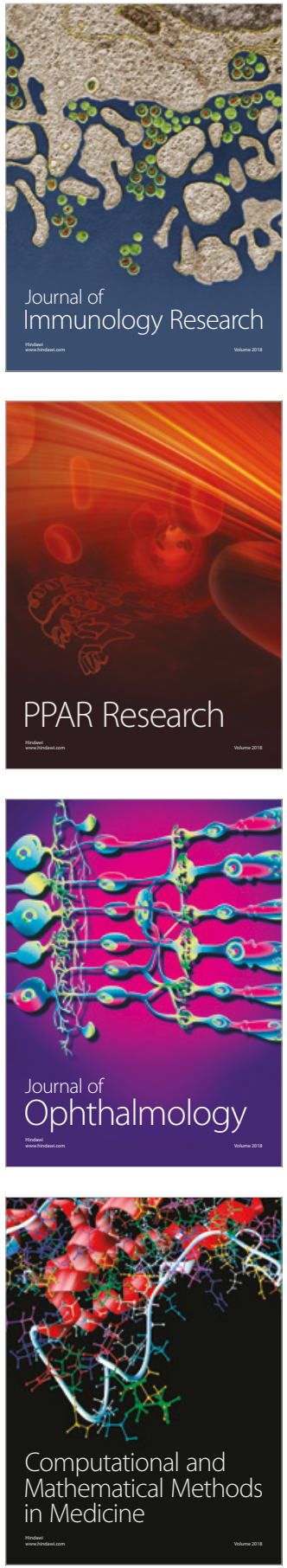

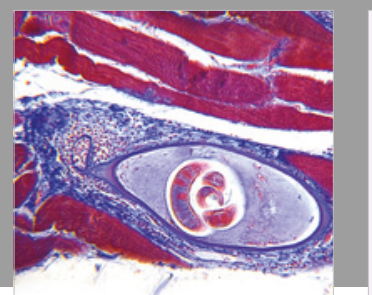

Gastroenterology Research and Practice

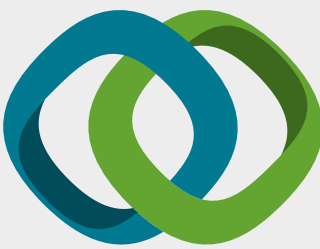

\section{Hindawi}

Submit your manuscripts at

www.hindawi.com
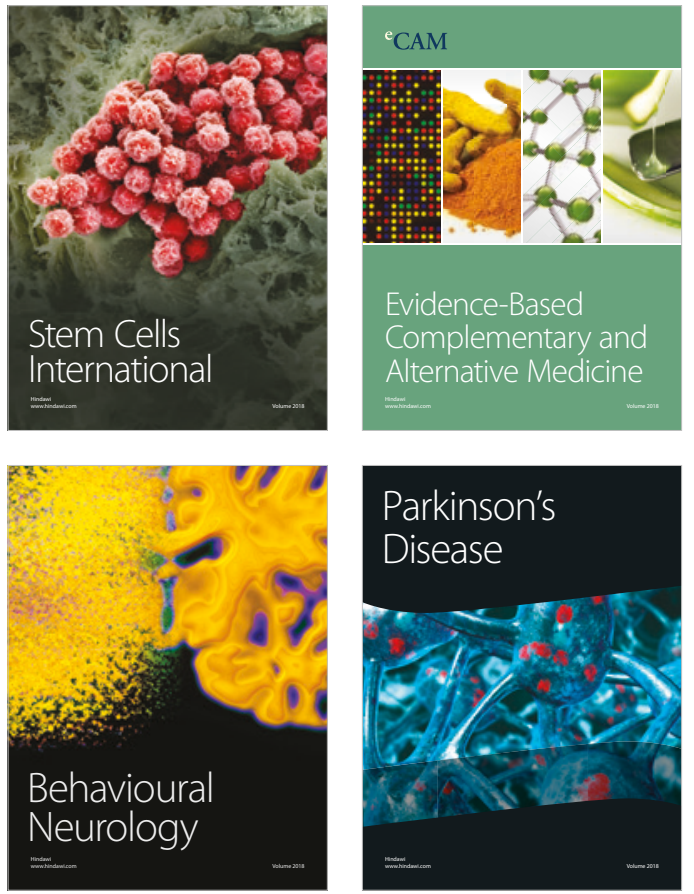

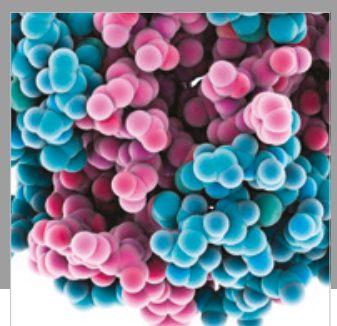

ournal of

Diabetes Research

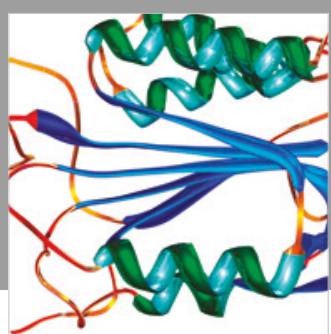

Disease Markers
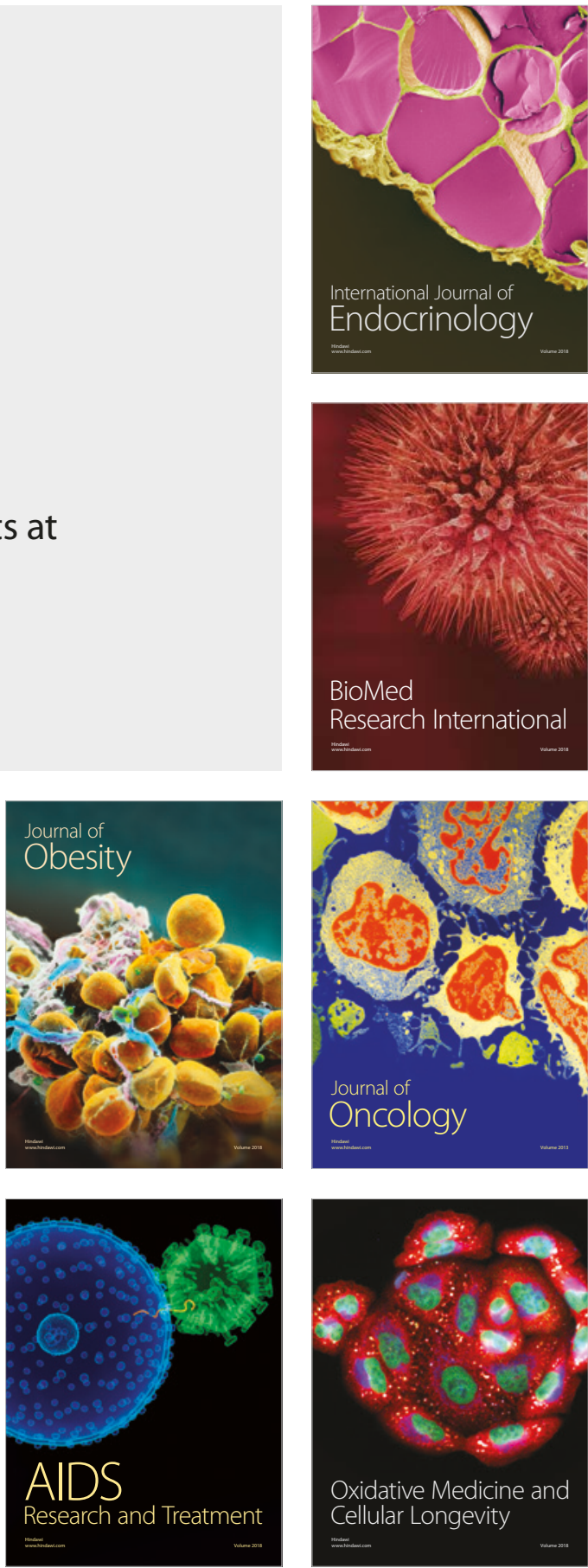